\title{
Alleviation of Oxidative Damage and Involvement of Nrf2-ARE Pathway in Mesodopaminergic System and Hippocampus of Status Epilepticus Rats Pretreated by Intranasal Pentoxifylline
}

\author{
Yunxiao Kang, ${ }^{1}$ Wensheng Yan, ${ }^{1,2}$ Hui Fang, ${ }^{1}$ Guoliang Zhang, ${ }^{3}$ Yakun Du, ${ }^{4}$ Lei Wang, \\ Huixian $\mathrm{Cui}^{3}{ }^{3}$ and Geming $\mathrm{Shi}^{1}$ \\ ${ }^{1}$ Department of Neurobiology, Hebei Medical University, Shijiazhuang, Hebei 050017, China \\ ${ }^{2}$ Department of Human Anatomy, Shijiazhuang Medical College, Shijiazhuang, Hebei 050599, China \\ ${ }^{3}$ Department of Human Anatomy, Hebei Medical University, Shijiazhuang, Hebei 050017, China \\ ${ }^{4}$ Department of Neurology, Children's Hospital of Hebei Province, Shijiazhuang, Hebei 050017, China \\ Correspondence should be addressed to Geming Shi; shigeming@163.com
}

Received 3 December 2016; Revised 6 February 2017; Accepted 21 February 2017; Published 12 March 2017

Academic Editor: Luciano Saso

Copyright (C) 2017 Yunxiao Kang et al. This is an open access article distributed under the Creative Commons Attribution License, which permits unrestricted use, distribution, and reproduction in any medium, provided the original work is properly cited.

\begin{abstract}
The current studies were aimed at evaluating the efficacy of intranasal pentoxifylline (Ptx) pretreatment in protecting mesodopaminergic system and hippocampus from oxidative damage of lithium-pilocarpine induced status epilepticus (SE) and the involvement of nuclear factor erythroid 2-related factor 2- (Nrf2-) antioxidant response elements pathway. Pentoxifylline was administered to rats intranasally or intraperitoneally 30 minutes before inducing SE. Our results showed the impaired visuospatial memory, the defected mesodopaminergic system, and the oxidative damage and the transient activation of Nrf2 in SE rats. The transient activation of Nrf2 in SE rats was enhanced by Ptx pretreatment, which was followed by the upregulation of heme oxygenase-1 and $\mathrm{NAD}(\mathrm{P}) \mathrm{H}$ :quinone oxidoreductase-1. Ptx pretreatment to SE rats significantly suppressed the epileptic seizures, decreased the levels of lipid peroxide and malondialdehyde, and elevated the ratio of reduced glutathione/oxidized glutathione. Compared with intraperitoneal injection, intranasal Ptx delivery completely restored the visuospatial memory and the activity of mesodopaminergic system in SE rats. Intranasal administration of Ptx may hopefully become a noninvasive, painless, and easily administered option for epileptic patients.
\end{abstract}

\section{Introduction}

Epilepsy is a life-threatening medical emergency that warrants immediate treatment to prevent seizure activity and associated neuronal damage [1]. Status epilepticus (SE) is one of the most severe conditions of epilepsy [2]. Patients with SE are at risk of neurologic complications. Early intervention is frequently needed to shorten seizure duration [3]. The sooner seizures are treated, the more likely they will be controlled [4]. However, intramuscular or intravenous injection cannot be implemented immediately outside of hospital settings, thus delaying or missing the best treatment time and threatening the human life. The intranasal route to administer drugs is quick and effective in targeting the brain $[4,5]$ and potentially provides a direct delivery of the drug to the central nervous system $[6,7]$ bypassing the blood-brain barrier and exerting therapeutic effects at terminating epileptic seizures. Intranasal drug administration is noninvasive, painless, and easily administered for epileptic patients [4] in the home treatment of prolonged seizures and in the treatment of prehospital seizures by emergency medical technicians [8].

The first-line drugs to stop seizures are benzodiazepines [9], which manipulate $\gamma$-aminobutyric acid receptors and make neurons resistant to excitation. In addition to controlling neuronal hyperactivity and excitotoxicity, one important factor that should be dealt with is oxidative stress in epileptic seizures [10-12]. Oxidative stress means an imbalance between oxidation and antioxidation in vivo, which leads to excessive oxygen free radical and reduced antioxidative capacity [13-15]. Excessive oxygen free radical generated 
in SE dramatically impairs the structure and function of neurons [16-18]. Nuclear factor erythroid 2-related factor 2 (Nrf2), as a transcription factor, controls the basal and inducible expression of an array of antioxidant and detoxification enzymes to degrade oxygen free radical $[13,19,20]$. Disruption of Nrf2-antioxidant response elements (ARE) pathway results in an increased susceptibility to oxidative insults and other toxicants [21]. Activation of Nrf2-ARE pathway protects neurons against oxidative damage and excitotoxic damage [13, 22-24].

Pentoxifylline (Ptx), a potent antioxidant and modulator of a variety of transmitters, was initially introduced for the treatment of respiratory and peripheral circulatory disorders. Currently, a beneficial effect of Ptx is found on preventing epileptic seizures $[25,26]$. The frequency and severity of epileptic seizures are ameliorated and oxidative damage is attenuated in Ptx-treated SE rats [25, 26]. Clinical and experimental studies have suggested the implication of dopaminergic system in seizures [27], such as a decreased DA content in hippocampus (Hip) and striatum of SE rats $[26,28]$, as well as the reduced dopamine transporter (DAT) in substantia nigra (SN) and midbrain of epilepsy patients $[29,30]$. Therefore, in the present study, the efficacy of intranasal Ptx delivery in epileptic seizures was investigated by analyzing the alteration of mesodopaminergic system and hippocampus in SE rats induced by lithium-pilocarpine (LiPc), based on the severe implication of mesodopaminergic system and Hip in SE [26, 29]. Meanwhile, oxidative stress parameters and Nrf2-ARE pathway were examined to evaluate whether Nrf2 was involved in the effects of Ptx treatment on mesodopaminergic system and hippocampus of SE rats. For comparison purposes, the same observations were performed in SE rats that experienced intraperitoneal Ptx treatment.

\section{Materials and Methods}

2.1. Animals and Housing. Three-month-old male Sprague Dawley rats were supplied by the Experimental Animal Center of Hebei Medical University and were housed under controlled conditions with 12-hour light-dark diurnal cycle at $22 \pm 2{ }^{\circ} \mathrm{C}$, with humidity at $50-60 \%$ and with free access to food and water. The experimental procedures followed the rules in the "Guidelines for the Care and Use of Mammals in Neuroscience and Behavioral Research" and were approved by the Committee of Ethics on Animal Experiments at Hebei Medical University.

2.2. Ptx Treatment and Induction of SE. Ptx was administered to rats either via nostril instillation or by intraperitoneal injection. For intranasal experiment, experimental rats were assigned to the following groups: CON-in $(n=25)$, LICI-in $(n=25)$, PTX-in $(n=25)$, SE-in $(n=35)$, and PTX.in-SE $(n=35)$. The rats in PTX-in and PTX.in-SE were intranasally given Ptx. Intranasal Ptx delivery was performed as described [31]. Briefly, fully conscious rats were held and laid upside down. The solution of Ptx (prepared in saline) was introduced by the pressure with a micropipette into one nasal cavity, without introducing the pipette tip directly into the nasal cavity, and the rats were immobilized in this position for $15 \mathrm{~s}$ by gently pulling the tail to prevent sneezing. The rats were discarded if sneezing happened. The same procedure was repeated in the other nostril. For intraperitoneal injection experiment, rats were divided into five groups consisting of CON-ip $(n=25)$, LICI-ip $(n=25)$, PTX-ip $(n=25)$, SE-ip $(n=35)$, and PTX.ip-SE $(n=35)$. The rats in PTXip and PTX.ip-SE received intraperitoneal Ptx injection. SE was induced in SE-in, SE-ip, PTX.in-SE, and PTX.ip-SE rats by administering an aqueous solution of lithium chloride (Li, $127 \mathrm{mg} / \mathrm{kg}, \mathrm{BDH}$ Laboratory Supplies) intraperitoneally, followed (20 hrs later) by injecting pilocarpine hydrochloride (Pc, $20 \mathrm{mg} / \mathrm{kg}$, Sigma) subcutaneously. Ptx was administered intranasally or intraperitoneally at the dose of $60 \mathrm{mg} / \mathrm{kg}$ (30 minutes before Pc injection) to rats corresponding to PTX.inSE and PTX.ip-SE, respectively. The rats in CON-in, LICIin, and PTX-in as well as CON-ip, LICI-ip, and PTX-ip received saline, lithium chloride, and Ptx (60 mg/kg, Sigma) correspondingly. After Pc injections, the rats were observed for the signs of seizure activity. Based on Racine's scale [32], the rats that showed consecutive seizures with a score of 3 or above fell in SE $[25,32]$. The latency and incidence of seizures as well as mortality within $24 \mathrm{hrs}$ were recorded. The rats were sacrificed $24 \mathrm{hrs}$ (for oxidative parameters and Nrf2ARE pathway) or 7 days (for Morris water maze test and parameters of mesodopaminergic system as well as oxidative parameters and Nrf2-ARE pathway) following Pc treatment. For the rats that were treated similarly in the groups CON-in (and ip), LICL-in (and ip), or SE-in (and ip), since the parameters were not substantially different (ANOVA), the data collected from them in the two experiments were clustered (cl) for analysis as CON-cl, LICL-cl, or SE-cl correspondingly, except for the data collected by Western blot.

2.3. Morris Water Maze Test. The rats in each group were tested for visuospatial memory $24 \mathrm{hrs}$ after the Pc injection using Morris water maze test as described previously [24]. The water maze included a circular water tank $(180 \mathrm{~cm}$ in diameter, $80 \mathrm{~cm}$ high) that was partially filled with water $(23 \pm$ $\left.1^{\circ} \mathrm{C}\right)$. The water was made opaque by adding milk to prevent visualization of the platform. The pool was divided virtually into four equal quadrants. A colorless escape platform $(10 \mathrm{~cm}$ in diameter) was hidden $1 \mathrm{~cm}$ below the surface of the water in a fixed location. The maze was located in a quiet room, surrounded by visual cues outside of the maze, which was used by the rats for spatial orientation. The experiments were conducted two sessions per day for 5 consecutive days, each session including four trials, with an intertrial interval of $60 \mathrm{~s}$ and an intersession interval of $2 \mathrm{hrs}$. In each trial, the animals were gently placed in the middle of the circular edge in a randomly selected quadrant, with the nose pointing toward the wall. If animals failed to find the escape platform within $120 \mathrm{~s}$ by themselves, they were placed on the platform for $10 \mathrm{~s}$ by the experimenter and their escape latency was accepted as $120 \mathrm{~s}$. After climbing onto the platform, the animal remained there for $30 \mathrm{~s}$ before the commencement of the next trial. On the sixth day, a probe trial without the platform was assessed, and the time spent in the target quadrant where the platform had been located was recorded. 
TABLE 1: Validation parameters of the LC-MS/MS method.

\begin{tabular}{lccccc}
\hline Analyte & $r$ & LLOQ $(\mathrm{ng} / \mathrm{g})$ & Recovery $(\%)$ & Intraprecision $(\mathrm{RSD} \%)$ & Interprecision $(\mathrm{RSD} \%)$ \\
\hline DA & 0.9969 & 2.0 & $94.6 \pm 8.7$ & 10.3 & 12.4 \\
DOPAC & 0.9982 & 72.0 & $93.4 \pm 6.6$ & 8.9 & 7.5 \\
HVA & 0.9977 & 50.0 & $95.3 \pm 7.4$ & 7.7 & 11.3 \\
\hline
\end{tabular}

DA: dopamine; DOPAC: 3,4-dihydroxyphenylacetic acid; HVA: homovanillic acid.

2.4. Sample Preparation. For biochemical, real-time quantitative PCR (qPCR) and Western blot analyses, the rats in each group were sacrificed by decapitation. The brains were removed quickly. The tissue block containing substantia nigra and ventral tegmental area (SN-VTA; between $3.00 \mathrm{~mm}$ and $4.08 \mathrm{~mm}$ ) or caudate putamen $(\mathrm{CPu}$; between $8.64 \mathrm{~mm}$ and $10.08 \mathrm{~mm}$ ) and Hip (between $5.40 \mathrm{~mm}$ and $6.08 \mathrm{~mm}$ ) relatively rostral to the interaural axis was dissected on icecold plate using a scalpel for ophthalmic surgery and a stereomicroscopy. The tissue blocks of rats in each group were processed for Western blot or liquid chromatography coupled with tandem mass spectrometry (LC-MS/MS) assay and the tissue blocks of others were chosen for lipid peroxide (LPO), malondialdehyde (MDA), reduced glutathione (GSH), and oxidized glutathione (GSSG) assay by spectrophotometry or prepared for qPCR analysis based on the study purposes.

2.5. Biochemical Analysis. For LPO, MDA, GSH, and GSSG assay, SN-VTA or Hip tissue block was weighed and homogenized with 10 times (w/v) ice-cold $0.1 \mathrm{M}$ phosphate buffer, $\mathrm{PH}$ 7.4. The homogenates were used to assess LPO, MDA, GSH, and GSSG spectrophotometrically using detection kits following the manufacturer's instruction (Nanjing Jiancheng Bioengineering Institute, China).

For dopamine (DA) and metabolites assay, $\mathrm{CPu}$ or Hip tissue block was weighed and homogenized in $80 \%$ acetonitrile containing $0.1 \%$ formic acid $(5 \mu \mathrm{L} / \mathrm{mg})$. The homogenates were centrifuged at $14,000 \mathrm{rpm}$ for $10 \mathrm{~min}$ at $4^{\circ} \mathrm{C}$. The supernatants were collected and stored at $-80^{\circ} \mathrm{C}$. DA, 3,4-dihydroxyphenylacetic acid (DOPAC), and homovanillic acid (HVA) were determined by LC-MS/MS. The LC separation was performed on Agilent 1200 LC system (Agilent, Santa Clara, USA) using a Synergi Fusion-RP C18 column $(50 \mathrm{~mm} \times 3.0 \mathrm{~mm}, 4 \mu \mathrm{m})$ provided by Phenomenex. MS/MS detection was carried out using a 3200 QTRAP $^{\mathrm{TM}}$ LC-MS/MS System (Applied Biosystems, Foster City, CA, USA). The multiple-reaction monitoring mode was used for the quantification. The principal validation parameters of the LCMS/MS were set up as shown in Table 1, based on the previous study [33].

2.6. $q$ PCR. $2 \mu \mathrm{g}$ of total RNA from the SN-VTA or Hip tissue block was subjected to reverse transcription using random primer to obtain the first-strand cDNA template. qPCR was performed with $0.8 \mu \mathrm{L}$ cDNA (diluted 1:10), specific primers $2 \mu \mathrm{L}$, and $2 \times \mathrm{GoTaq}^{\circledR}$ Green Master Mix (Promega, USA) with a final volume of $20 \mu \mathrm{L}$. PCR was performed as follows: an initial cycle at $95^{\circ} \mathrm{C}$ for $10 \mathrm{~min}$, followed by 40 cycles at $95^{\circ} \mathrm{C}$ for $15 \mathrm{~s}, 58^{\circ} \mathrm{C}$ for $20 \mathrm{~s}$, and $72^{\circ} \mathrm{C}$ for $27 \mathrm{~s}$. Then PCR products were analyzed by melting curve to confirm the specificity of amplification. Expression of tyrosine hydroxylase (TH), dopamine transporter (DAT), Nrf2, heme oxygenase-1 (HO1) and $\mathrm{NAD}(\mathrm{P}) \mathrm{H}$ :quinone oxidoreductase-1 (NQO-1) genes was detected. The relative quantification was calculated using the $2^{-\Delta \Delta \text { ct }}$ method. GAPDH was used as reference gene in all calculations. The sets of primers were as follows: TH (5'-GCTTCTCTGACCAGGTGTATCG- $3^{\prime}$ and $5^{\prime}$-GCAATCTCTTCCGCTGTGTAT- $\left.3^{\prime}\right)$, DAT (5'-ACTCTGTGAGGCATCTGTGTG-3' and 5'-TGTAACTGGAGAAGGCAATCAG-3 $\left.{ }^{\prime}\right)$, Nrf2 (5'-GACCTAAAGCACAGCCAACACAT- $3^{\prime}$ and $5^{\prime}$-CTCAATCGGCTTGAATGTTTGTC$\left.3^{\prime}\right)$, HO-1 (5' -TGTCCCAGGATTTGTCCGAG-3' and $5^{\prime}$ ACTGGGTTCTGCTTGTTTCGCT-3), NQO-1 (5'-GGGGACATGAACGTCATTCTCT- $3^{\prime}$ and $5^{\prime}$-AGTGGTGACTCCTCCCAGACAG- $3^{\prime}$ ), and GAPDH ( $5^{\prime}$-TGAACGGGAAGCTCACTG- $3^{\prime}$ and $5^{\prime}$-GCTTCACCACCTTCTTGATG$\left.3^{\prime}\right)$.

2.7. Western Blot Analysis. For detection of TH and DAT protein levels, SN-VTA, $\mathrm{CPu}$, or Hip tissue block was homogenized in Radioimmunoprecipitation Assay (RIPA) buffer containing $1 \%$ Triton X-100, $0.1 \%$ SDS, $0.5 \%$ sodium deoxycholate, and protease inhibitors (phenylmethanesulfonyl fluoride $100 \mu \mathrm{g} / \mathrm{mL}$, aprotinin $30 \mu \mathrm{g} / \mathrm{mL}$, and sodium orthovanadate $1 \mathrm{mM}$ ) and then sonicated for $4 \times 10 \mathrm{~s}$. After centrifugation at $12,000 \times \mathrm{g}$ for $20 \mathrm{~min}$ at $4^{\circ} \mathrm{C}$, the supernatant was collected and centrifuged again as above. The final resulting supernatant was stored at $-80^{\circ} \mathrm{C}$ until use. Samples from SN-VTA, CPu, or Hip were diluted in 2x sample buffer (50 mM Tris, pH 6.8, 2\% SDS, 10\% glycerol, $0.1 \%$ bromophenol blue, and $5 \% \beta$-mercaptoethanol) and heated for $5 \mathrm{~min}$ at $95^{\circ} \mathrm{C}$ before SDS-PAGE on a $10 \%$ gel and subsequently transferred to a PVDF membrane. The membrane was incubated for $2 \mathrm{~h}$ with $5 \%$ nonfat dry milk in Tris-buffered saline (TBS) containing 0.05\% Tween-20 (TBST) $(20 \mathrm{mM}$ Tris-Cl, $137 \mathrm{mM} \mathrm{NaCl}, 0.1 \%$ Tween 20, $\mathrm{pH}$ 7.6) at room temperature. The membrane was rinsed in three changes of TBST and then incubated overnight with mouse anti-TH monoclonal antibody $(1: 10,000$, Sigma) or rabbit anti-DAT polyclonal antibody $\left(1: 4000\right.$, Millipore) at $4^{\circ} \mathrm{C}$. After three washes, the membrane was incubated for $1 \mathrm{~h}$ in IRDye ${ }^{\circledR}$ 800 -conjugated goat anti-mouse second antibody $(1: 3000$, Rockland) or goat anti-rabbit second antibody (1:3000, Rockland) at room temperature. The relative density of bands was analyzed on an Odyssey infrared scanner (LI-COR Biosciences). Following stripping, each PVDF membrane was subsequently immunoblotted with mouse anti- $\beta$-actin monoclonal antibody (1:6000, Santa Cruz Biotechnology). The labeling densities for TH or DAT were compared with those of $\beta$-actin, which were the endogenous control. 


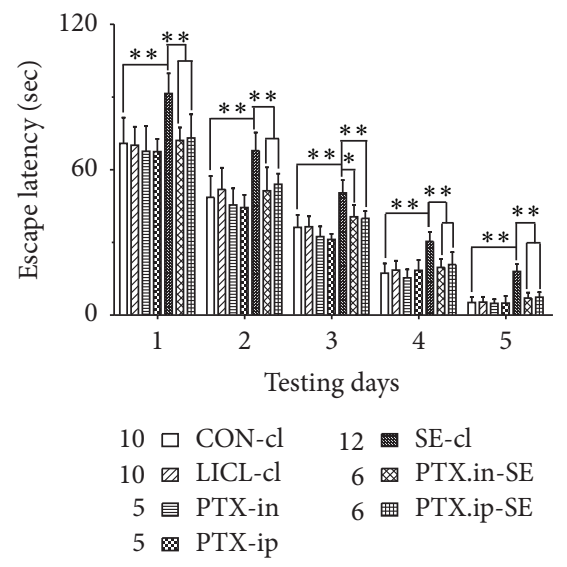

(a)

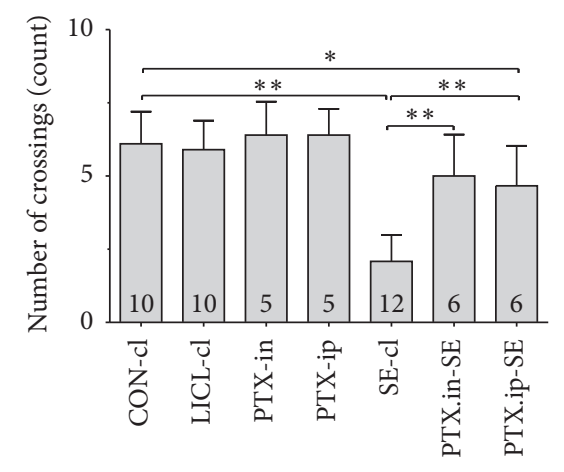

(b)

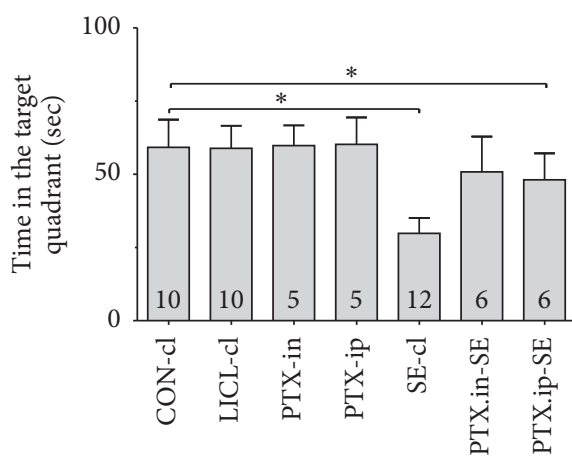

(c)

FIGURE 1: Effects of intranasal and intraperitoneal Ptx pretreatment on the visuospatial memory of SE rats induced by Li-Pc. (a) The escape latency to reach the platform. (b) The number of crossings. (c) The time spent in the target quadrant. The results were expressed as the means \pm SD. ${ }^{*} P<0.05 ;{ }^{* *} P<0.01$.

For detection of Nrf2, HO-1, or NQO-1 protein levels, SN-VTA or Hip tissue block was homogenized in ice-cold lysis buffer $(10 \mathrm{mmol} / \mathrm{L}$ HEPES, pH 7.9, $10 \mathrm{mmol} / \mathrm{L} \mathrm{KCl}$, $0.1 \mathrm{mmol} / \mathrm{L} \mathrm{EDTA}, 1 \mathrm{mmol} / \mathrm{L} \mathrm{DTT}$, and $0.1 \mathrm{mmol} / \mathrm{L}$ EGTA) for $15 \mathrm{~min}$. After adding NP-40, the homogenate was centrifuged at $10,000 \mathrm{rpm}$ at $4^{\circ} \mathrm{C}$ for $3 \mathrm{~min}$ and the supernatant was collected as cytoplasmic protein for HO-1 and NQO1. The pellets were homogenized in ice-cold lysis buffer $(20 \mathrm{mmol} / \mathrm{L}$ HEPES, pH $7.9,400 \mathrm{mmol} / \mathrm{L} \mathrm{NaCl}, 1 \mathrm{mmol} / \mathrm{L}$ EDTA, and $0.1 \mathrm{mmol} / \mathrm{L}$ EGTA) for $15 \mathrm{~min}$. Then the pellets were centrifuged at $12,000 \mathrm{rpm}$ at $4^{\circ} \mathrm{C}$ for $10 \mathrm{~min}$, and the supernatant was collected. Phenylmethanesulfonyl fluoride was added to the supernatant with the final concentration of $1 \mathrm{mmol} / \mathrm{L}$ as the nuclear protein for Nrf2. Samples from SN-VTA or Hip $(50 \mu \mathrm{g})$ were separated by SDS/PAGE and transferred onto PVDF membranes. Membranes were blocked with 5\% skimmed milk for $1 \mathrm{~h}$ at room temperature and then were probed with polyclonal rabbit antiNrf2 antibody (1:500, Abcam), polyclonal rabbit anti-HO1 antibody $(1: 200$, Abcam), or polyclonal rabbit anti-NQO1 antibody $(1: 200, \mathrm{Abcam})$ overnight at $4^{\circ} \mathrm{C}$. After washing three times with phosphate buffered saline with $1 \%$ Tween 20, IRDye 800-conjugated goat anti-rabbit second antibody (1:3000, Rockland) incubated with membranes for $1 \mathrm{~h}$ at room temperature. The relative density of bands was analyzed on an Odyssey infrared scanner (LI-COR Biosciences). The densitometry values were normalized with respect to the values of anti-histone 3 for Nrf2 or anti- $\beta$-actin for $\mathrm{HO}-1$ and NQO-1 immunoreactivity.

2.8. Statistical Analysis. Measurement data were described with mean \pm SD. Levene's test was applied to test homogeneity of variance. If both normal distribution $(P>0.1)$ and homogeneity of variance $(P>0.1)$ were found, then parametric test was performed by one-way analysis of variance (one-way ANOVA) followed by a Student-Newman-Keuls (SNK) post hoc test for multiple comparisons. Otherwise, nonparametric statistics were done by Kruskal-Wallis test followed by Mann-Whitney $U$ test for post hoc analysis between groups. A difference was considered statistically significant at a $P$ value of less than 0.05 .

\section{Results}

3.1. Antiepileptic Effects of Ptx Pretreatment. The effects of intranasal Ptx pretreatment on epileptic activities were analyzed. All the rats in SE-cl developed into epileptic seizures and then into SE after the injection of Pc. 10\% of them died over a period of $24 \mathrm{hrs}$. Ptx pretreatment significantly extended the latency to first seizure and decreased epileptic seizures. $17.14 \%$ of rats in PTX.in-SE and $20 \%$ of rats in PTX.ip-SE developed into seizures. No rats in PTX.inSE and PTX.ip-SE developed into SE and died (Table 2). Intranasal Ptx administration lessened epileptic activities as intraperitoneal injection of Ptx did and had antiepileptic effects.

3.2. Visuospatial Memory. Behavioral parameters in water maze test were analyzed to reveal the effects of intranasal Ptx pretreatment on visuospatial memory of SE rats. Group differences in the escape latency (Figure 1(a), $1 \mathrm{~d}: \chi^{2}=23.338$ and $P<0.01 ; 2 \mathrm{~d}: \chi^{2}=26.167$ and $P<0.01 ; 3 \mathrm{~d}: F(6,47)=$ 17.824 and $P<0.01 ; 4 \mathrm{~d}: \chi^{2}=30.381$ and $P<0.01$; $5 \mathrm{~d}: \chi^{2}=32.735$ and $\left.P<0.01\right)$, the number of crossings (Figure $1(\mathrm{~b}), \chi^{2}=33.250$ and $P<0.01$ ), and the time in the target quadrant (Figure $1(\mathrm{c}), \chi^{2}=32.996$ and $P<0.01$ ) were found among the CON-cl, LICL-cl, PTX-in, PTX-ip, SE-cl, PTX.in-SE, and PTX.ip-SE rats. The post hoc test showed that the rats in SE-cl exhibited the longer escape latency to reach the platform $(P<0.01)$, the reduced number of crossings $(P<0.01)$, and the less time in the target quadrant $(P<0.05)$, compared with rats in CON-cl. Intranasal or intraperitoneal Ptx pretreatment to SE rats shortened the escape latency to reach the platform $(P<0.05)$ and increased the number of crossings and the time spent in the target quadrant 
TABLE 2: Effects of pentoxifylline pretreatment on status epilepticus rats.

\begin{tabular}{|c|c|c|c|c|c|}
\hline Group & $n$ & Seizures (\%) & Latency to first seizure (min) & SE (\%) & Mortality (\%) within $24 \mathrm{~h}$ \\
\hline $\mathrm{CON}-\mathrm{cl}$ & 50 & $0(0)$ & - & - & 0 \\
\hline LICL-cl & 50 & $0(0)$ & - & - & 0 \\
\hline PTX-in & 25 & $0(0)$ & - & - & 0 \\
\hline PTX-ip & 25 & $0(0)$ & - & - & 0 \\
\hline SE-cl & 70 & 100 & $13.87 \pm 4.73$ & 100 & 10 \\
\hline PTX.in-SE & 35 & $17.14^{*}$ & $37.78 \pm 6.25^{*}$ & 0 & 0 \\
\hline PTX.ip-SE & 35 & $20^{*}$ & $28.08 \pm 7.10^{*}$ & 0 & 0 \\
\hline
\end{tabular}

${ }^{*} P<0.01$ versus $S E$ group.

$(P<0.01)$. There were no differences in the behavioral parameters of rats between PTX.in-SE and PTX.ip-SE. The behavioral parameters of rats in PTX.in-SE were completely restored to CON-cl rats, compared with rats in PTX.ip-SE. Intranasal Ptx pretreatment ameliorated the poor visuospatial memory of SE rats.

3.3. Mesodopaminergic System. To reveal whether intranasal Ptx pretreatment ameliorated the mesodopaminergic activity in SE rats, the markers of mesodopaminergic system were analyzed.

3.3.1. DA and Its Metabolites. Group differences among CON-cl, LICL-cl, PTX-in, PTX-ip, SE-cl, PTX.in-SE, and PTX.ip-SE rats were observed in DA (CPu: $\chi^{2}=37.676$ and $P<0.01$; Hip: $F(6,47)=78.847$ and $P<0.01)$, DOPAC (CPu: $F(6,47)=45.800$ and $P<0.01$; Hip: $F(6,47)=$ 146.455 and $P<0.01)$, and HVA content $(\mathrm{CPu}: F(6,47)=$ 73.834 and $P<0.01$; Hip: $F(6,47)=55.916$ and $P<0.01)$ (Table 3$)$. The post hoc test revealed the decreased DA, DOPAC, and HVA in CPu and Hip of rats in SE-cl, compared with rats in $\mathrm{CON}-\mathrm{cl}(P<0.01)$. Intranasal or intraperitoneal Ptx pretreatment to SE rats increased DA and its metabolites in $\mathrm{CPu}$ and Hip $(P<0.01)$. No differences in DA and its metabolites were shown in $\mathrm{CPu}$ and Hip of rats between PTX.in-SE and PTX.ip-SE. Intranasal Ptx pretreatment completely restored DA and its metabolites of SE rats to the levels of CON-cl rats, compared with rats in PTX.ip-SE.

3.3.2. TH and DAT $m$ RNAs. Group differences in TH mRNA (Figure 2(a), $F(6,47)=62.738$ and $P<0.01$ ) and DAT mRNA (Figure 3(a), $\chi^{2}=37.814$ and $P<0.01$ ) were detected in SN-VTA of rats among CON-cl, LICL-cl, PTX-in, PTXip, SE-cl, PTX.in-SE, and PTX.ip-SE. The post hoc test found that the levels of TH and DAT mRNAs were lower in SE$\mathrm{cl}$ rats than in $\mathrm{CON}-\mathrm{cl}$ rats $(P<0.01)$. Intranasal or intraperitoneal Ptx pretreatment to SE rats significantly increased TH and DAT mRNAs in SN-VTA $(P<0.01)$. No differences in TH and DAT mRNAs were shown in SN-VTA between PTX.in-SE rats and PTX.ip-SE rats. Intranasal Ptx pretreatment completely restored $\mathrm{TH}$ and DAT mRNAs of SE rats to the levels of CON-cl rats, compared with rats in PTX.ip-SE.

3.3.3. TH and DAT Proteins. Western blot was used to reveal the protein levels of $\mathrm{TH}$ and DAT extracted from SN-VTA,
$\mathrm{CPu}$, and Hip. TH and DAT were located at approximately 60 and $80 \mathrm{kDa}$, respectively. The group differences among CON-in, LICL-in, PTX-in, SE-in, and PTX.in-SE rats were found in the expression of TH (Figures 2(b) and 2(d), SNVTA: $F(4,22)=144.914$ and $P<0.01$; Figure 2(e), $\mathrm{CPu}$ : $F(4,22)=50.721$ and $P<0.01$; Figure $2(\mathrm{f})$, Hip: $F(4,22)=$ 18.219 and $P<0.01$ ) and DAT (Figures 3(b) and 3(d), SNVTA: $\chi^{2}=14.314$ and $P<0.01$; Figure 3(e), CPu: $\chi^{2}=$ 16.289 and $P<0.01$; Figure 3(f), Hip: $\chi^{2}=17.015$ and $P<0.01$ ), as well as in that of TH (Figures 2(c), 2(g)-2(i); SN-VTA, CPu, and Hip: $P<0.01$ ) and DAT (Figures 3(c), 3(g)-3(i); SN-VTA, CPu, and Hip: $P<0.01$ ) among CON-ip, LICL-ip, PTX-ip, SE-ip, and PTX.ip-SE rats. The post hoc test revealed the decreased TH and DAT in SE-in rats and in SEip rats, compared with corresponding control rats $(P<0.01)$. Intranasal delivery, as well as intraperitoneal injection, of Ptx to $\mathrm{SE}$ rats elevated the levels of TH and DAT in SN-VTA, CPu, and Hip $(P<0.01)$. There were no differences in the levels of TH and DAT between PTX.in-SE rats and PTX.ip-SE rats. Intranasal Ptx pretreatment to SE rats completely restored $\mathrm{TH}$ and DAT proteins in SN-VTA, CPu, and Hip, compared with rats in PTX.ip-SE.

3.4. LPO, MDA, GSH, and GSSG. To assess whether the amelioratory effects of intranasal Ptx pretreatment to SE rats was associated with the oxidative stress, LPO, MDA, and the ratio of GSH/GSSG in SN-VTA and Hip were analyzed. There were group differences in LPO (Figure 4(a), SN-VTA (24 hrs): $\chi^{2}=32.447$ and $P<0.01$; SN-VTA (7 days): $\chi^{2}=33.837$ and $P<0.01$; Figure 4(b), Hip (24 hrs): $\chi^{2}=31.413$ and $P<0.01$; Hip (7 days): $\chi^{2}=36.309$ and $P<0.01$ ), MDA (Figure 4(c), SN-VTA (24 hrs): $\chi^{2}=33.813$ and $P<0.01$; SN-VTA (7 days): $\chi^{2}=35.623$ and $P<0.01$; Figure 4(d), Hip (24 hrs): $\chi^{2}=36.381$ and $P<0.01$; Hip (7 days): $\chi^{2}=26.853$ and $P<0.01$ ), and the ratio of GSH/GSSG (Figure 4(e), SN-VTA (24hrs): $\chi^{2}=38.609$ and $P<0.01$; SN-VTA $(7$ days): $\chi^{2}=30.277$ and $P<0.01$; Figure 4(f), Hip (24 hrs): $F(6,47)=42.160$ and $P<0.01$; Hip (7 days): $F(6,47)=$ 13.550 and $P<0.01)$ among the CON-cl, LICL-cl, PTX-in, PTX-ip, SE-cl, PTX.in-SE, and PTX.ip-SE rats. The post hoc test detected the increased LPO and MDA contents as well as the decreased ratio of GSH/GSSG in SN-VTA and Hip of SEcl rats, compared with $\mathrm{CON}-\mathrm{cl}$ rats $(P<0.01)$. The reduced levels of LPO and MDA and the elevated ratio of GSH/GSSG 


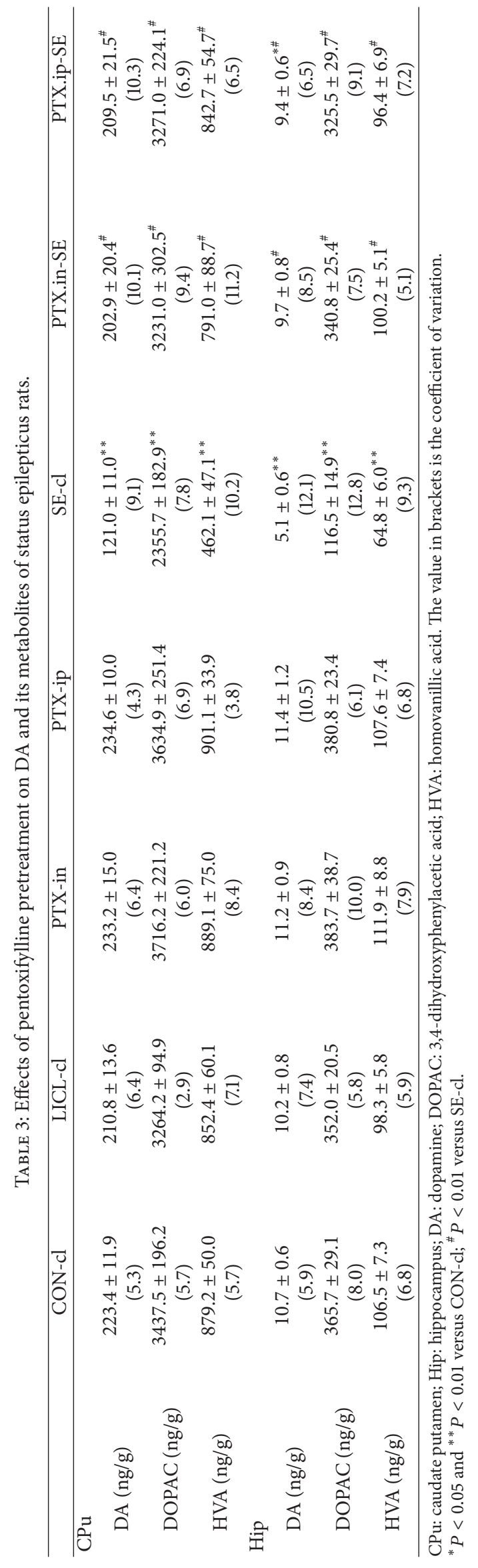




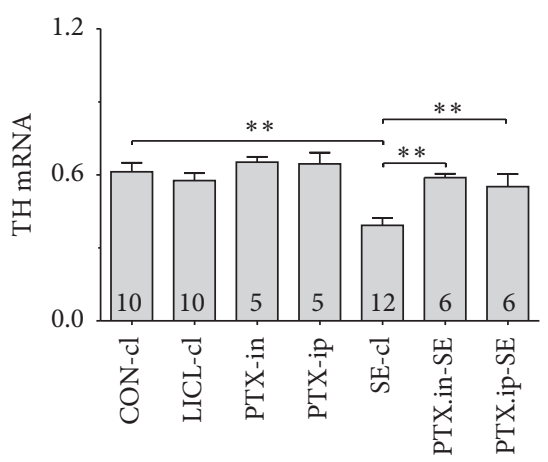

(a)

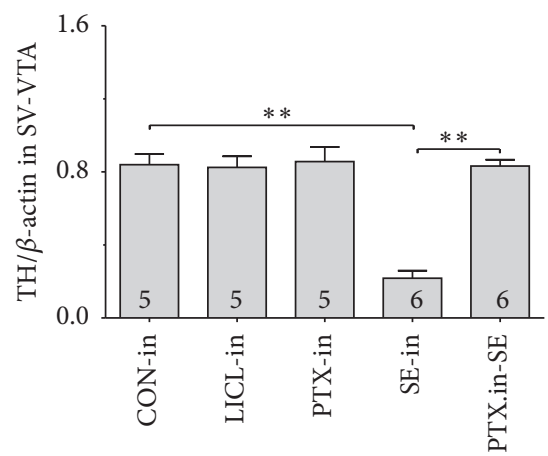

(d)

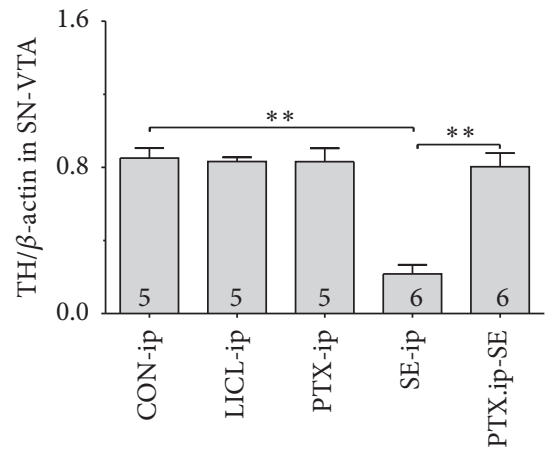

(g)

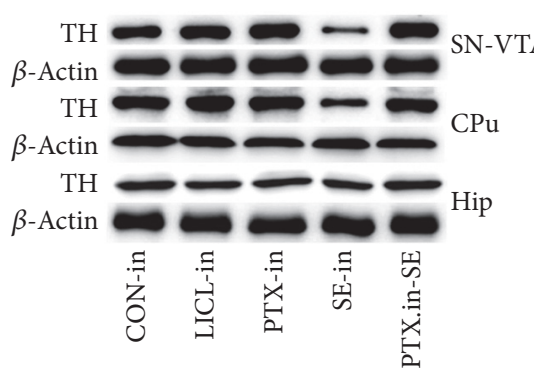

(b)

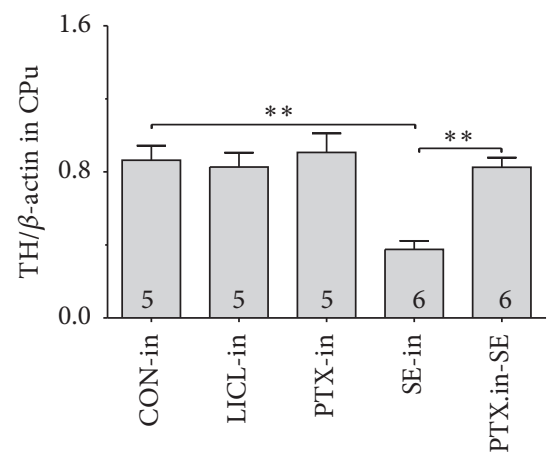

(e)

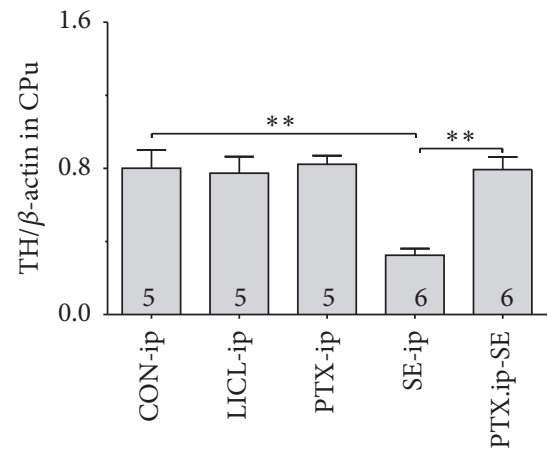

(h)

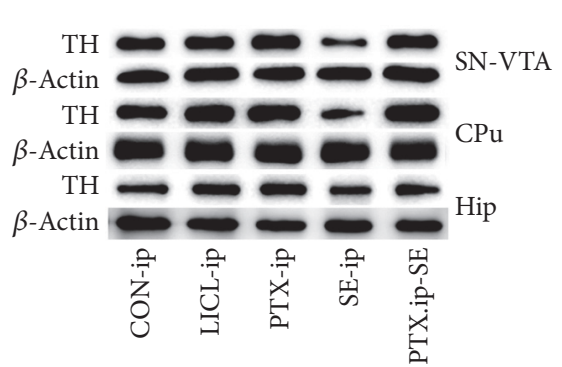

(c)

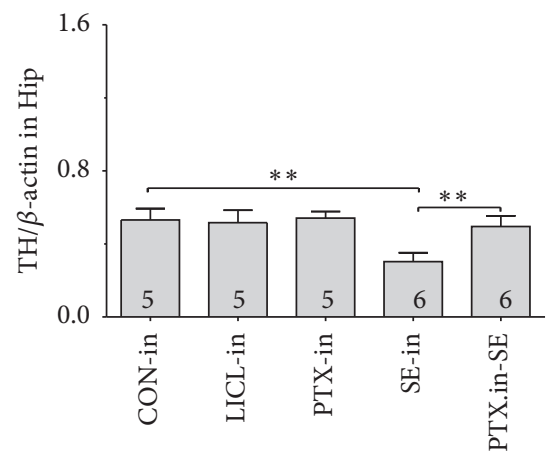

(f)

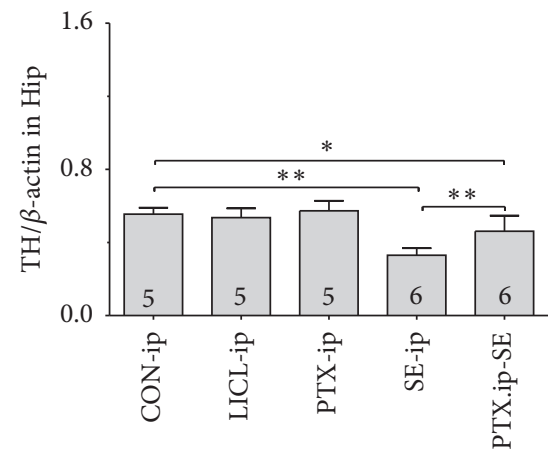

(i)

FIgURE 2: Effects of intranasal and intraperitoneal Ptx pretreatment on TH at mRNA and protein level of SE rats induced by Li-Pc. (a) TH mRNAs were detected in SN-VTA by qPCR. (b-i) TH protein was detected in SN-VTA, CPu, and Hip by Western blot. The results were expressed as the means $\pm \mathrm{SD} .{ }^{*} P<0.05 ;{ }^{* *} P<0.01$.

in SN-VTA and Hip were detected in rats of PTX.in-SE as well as PTX.ip-SE, compared with the rats in SE-cl $(P<0.01)$. Intranasal Ptx pretreatment to SE rats mitigated the oxidative damage in SN-VTA and Hip.

3.5. Nrf2-ARE. To evaluate whether the Nrf2-ARE pathway was involved in the alleviation of the oxidative damage in $\mathrm{SN}$ VTA and Hip of intranasal Ptx-pretreated SE rats, Nrf2, HO-1, and NQO-1 were examined at mRNA and protein levels.

3.5.1. Nrf2, HO-1 and NQO-1 mRNAs. Group differences among the CON-cl, LICL-cl, PTX-in, PTX-ip, SE-cl, PTX.inSE, and PTX.ip-SE rats were found in the levels of Nrf2, HO1, and NQO-1 mRNAs in SN-VTA (Figure 5(a), Nrf2 (24 hrs): $\chi^{2}=36.650$ and $P<0.01 ; \operatorname{Nrf} 2$ (7 days): $\chi^{2}=34.573$ and $P<$ 0.01; Figure 6(a), HO-1 (24 hrs): $\chi^{2}=37.430$ and $P<0.01$; HO-1 (7 days): $F(6,47)=28.400$ and $P<0.01$; Figure $7(\mathrm{a})$, NQO-1 (24 hrs): $F(6,47)=27.723$ and $P<0.01$; NQO-1 $(7$ days): $\chi^{2}=35.171$ and $P<0.01$ ), and Hip (Figure $8(\mathrm{a}), \mathrm{Nrf} 2$ (24 hrs): $\chi^{2}=35.997$ and $P<0.01 ; \operatorname{Nrf2}$ (7 days): $F(6,47)=$ 37.925 and $P<0.01$; Figure 9(a), HO-1 (24 hrs): $\chi^{2}=39.571$ and $P<0.01$; HO-1 (7 days): $\chi^{2}=36.662$ and $P<0.01$; Figure 10(a), NQO-1 (24 hrs): $F(6,47)=15.841$ and $P<0.01$; NQO-1 (7 days): $\chi^{2}=40.301$ and $\left.P<0.01\right)$. The post hoc test revealed the elevation of Nrf2, HO-1, and NQO-1 mRNAs in SN-VTA and in Hip of SE-cl rats 24 hrs following Pc injection $(P<0.01)$ and the significant reduction of them 7 days after Pc administration $(P<0.01)$, compared with $\mathrm{CON}-\mathrm{cl}$ rats. 


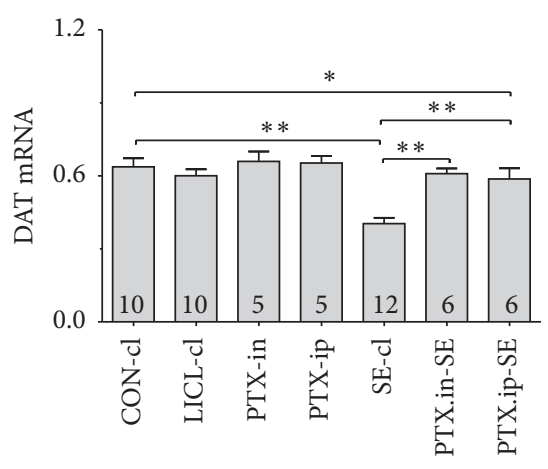

(a)

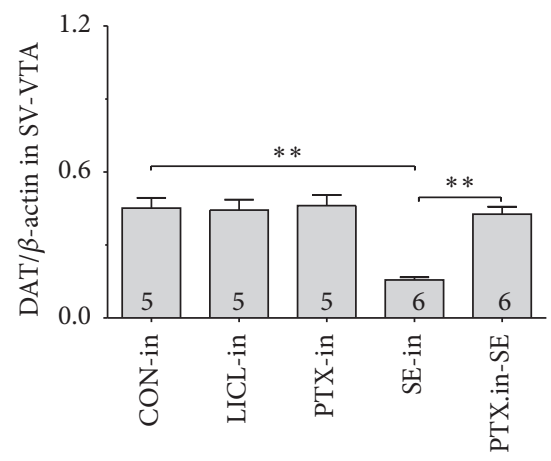

(d)

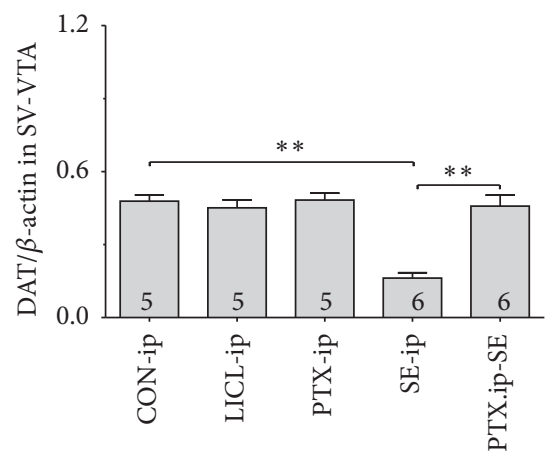

(g)

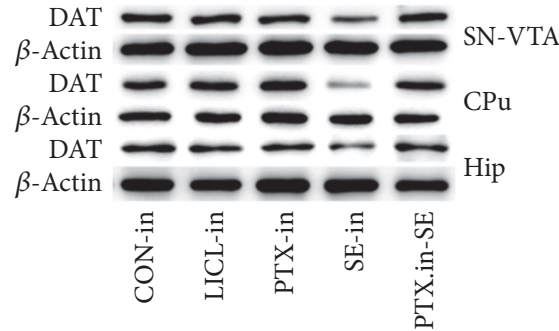

(b)

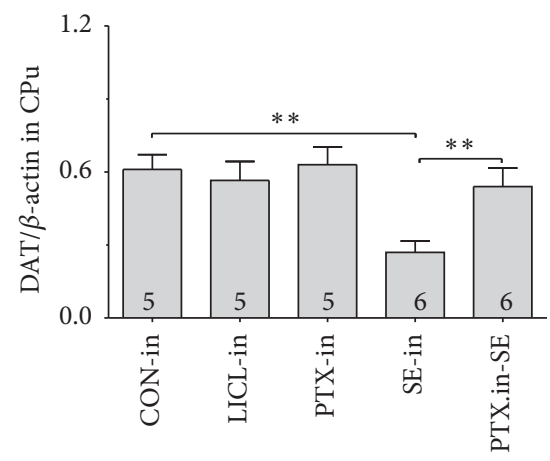

(e)

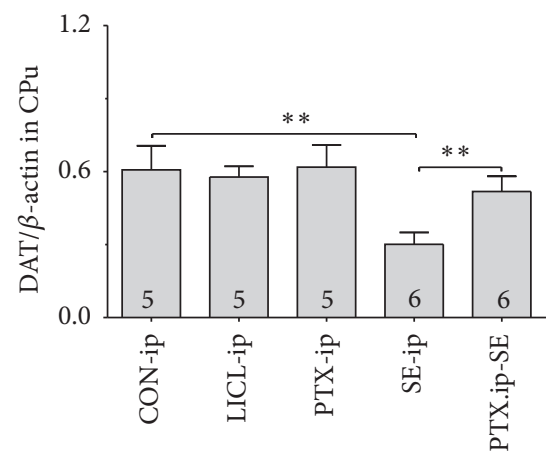

(h)

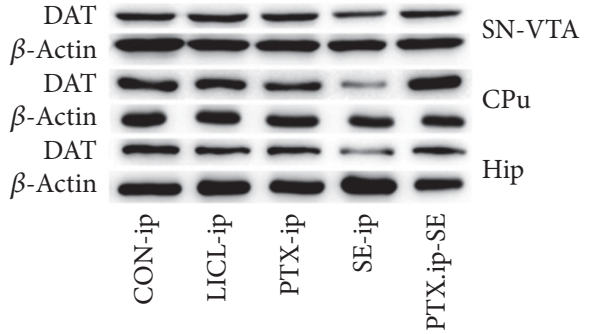

(c)

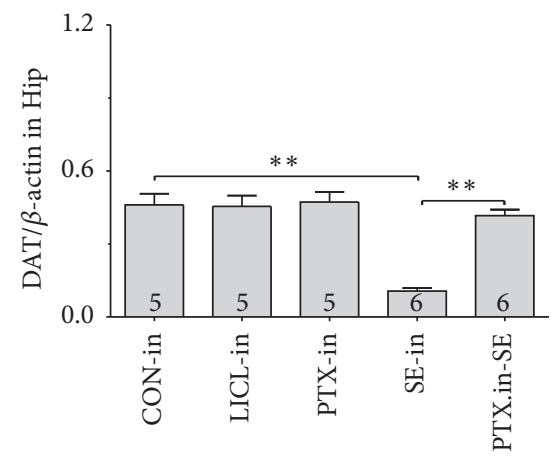

(f)

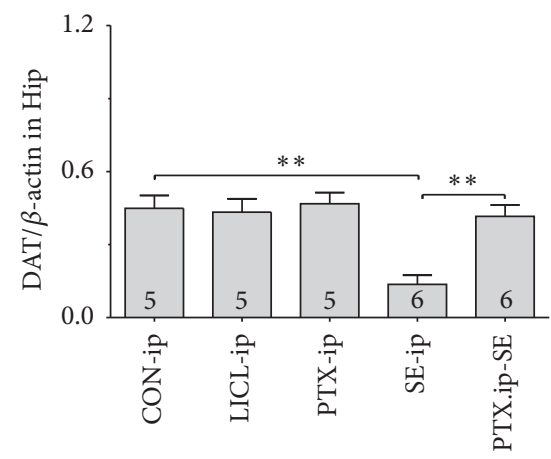

(i)

FIgure 3: Effects of intranasal and intraperitoneal Ptx pretreatment on DAT at mRNA and protein level of SE rats induced by Li-Pc. (a) DAT mRNA was detected in SN-VTA by qPCR. (b-i) DAT protein was detected in SN-VTA, CPu, and Hip by Western blot. The results were expressed as the means \pm SD. ${ }^{*} P<0.05 ;{ }^{* *} P<0.01$.

In SN-VTA, the increased Nrf2, HO-1, and NQO-1 mRNAs were detected in PTX.in-SE and PTX.ip-SE rats $(P<0.05)$, compared with SE-cl rats, except NQO-1 mRNA in PTX.ipSE rats $24 \mathrm{hrs}$ following Pc injection $(P=0.234)$. In Hip, the elevated Nrf2, HO-1, and NQO-1 mRNAs were observed only in PTX.in-SE and PTX.ip-SE rats 7 days following Pc injection, compared with SE-cl rats $(P<0.05)$.

3.5.2. Nrf2, HO-1, and NQO-1 Proteins. The protein levels of Nrf2, HO-1, and NQO-1 extracted from SN-VTA and Hip were detected by Western blot. Nrf2, HO-1, and NQO-1 were located at approximately 110,32 , and $31 \mathrm{kDa}$, respectively. The group differences among CON-in, LICL-in, PTX-in, SE-in, and PTX.in-SE rats were found in the expression of Nrf2,
HO-1, and NQO-1 in SN-VTA (Figures 5(b) and 5(c), Nrf2 (24 hrs): $F(4,22)=15.882$ and $P<0.01 ;$ Nrf2 (7 days): $F(4,22)=84.169$ and $P<0.01$; Figures $6(\mathrm{~b})$ and $6(\mathrm{c})$, HO-1 (24 hrs): $\chi^{2}=21.011$ and $P<0.01$; HO-1 (7 days): $F(4,22)=71.535$ and $P<0.01$; Figures $7(\mathrm{~b})$ and $7(\mathrm{c})$, NQO-1 (24hrs): $\chi^{2}=21.705$ and $P<0.01$; NQO-1 $(7$ days): $\chi^{2}=17.290$ and $P<0.01$ ), and Hip (Figures 8 (b) and 8(c), Nrf2 (24 hrs): $\chi^{2}=20.481$ and $P<0.01$; Nrf2 (7 days): $F(4,22)=27.035$ and $P<0.01$. Figures $9(\mathrm{~b})$ and $9(\mathrm{c})$, HO-1 (24 hrs): $\chi^{2}=19.504$ and $P<0.01$; HO-1 (7 days): $F(4,22)=78.424$ and $P<0.01$; Figures $10(\mathrm{~b})$ and $10(\mathrm{c})$, NQO-1 (24 hrs): $\chi^{2}=17.695$ and $P<0.01$; NQO-1 ( 7 days): $F(4,22)=67.737$ and $P<0.01)$, as well as in the levels of Nrf2 (Figures 5(b) and 5(d), SN-VTA; Figures 8(b) and 


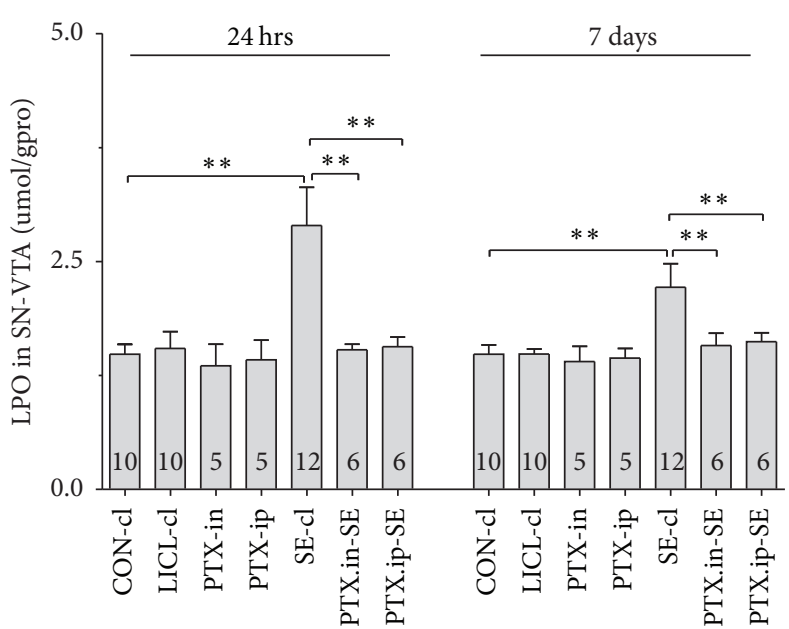

(a)

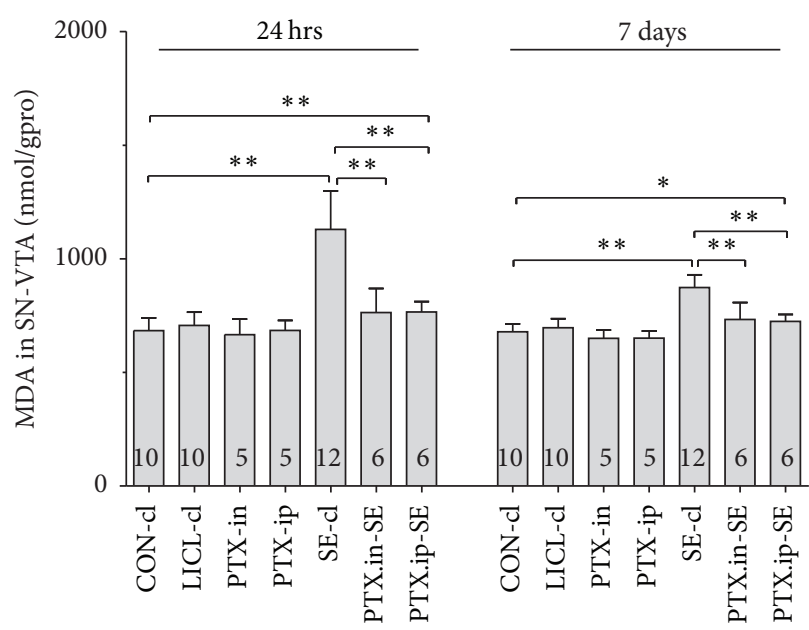

(c)

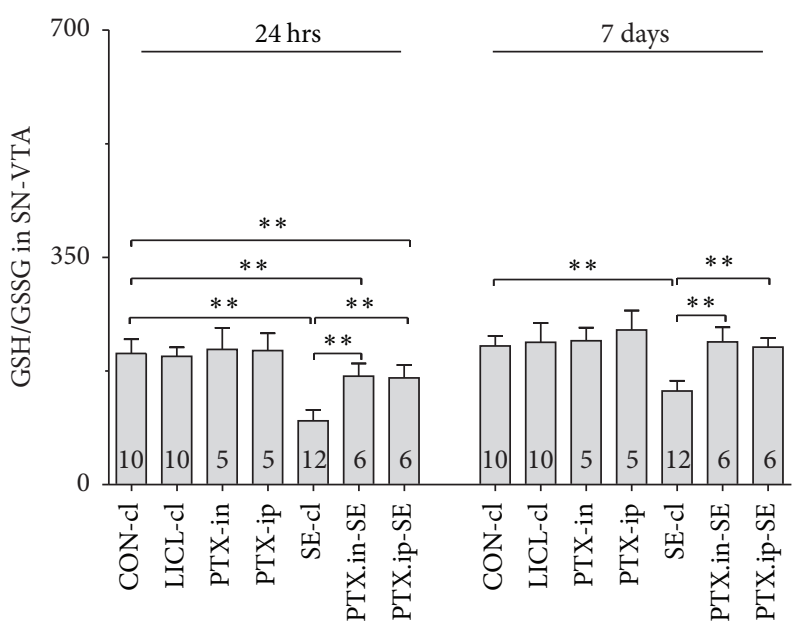

(e)

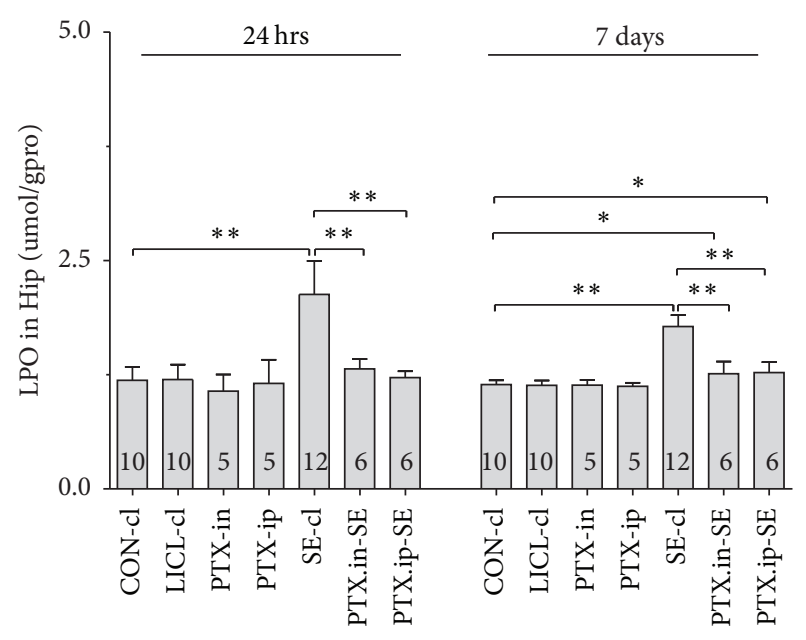

(b)

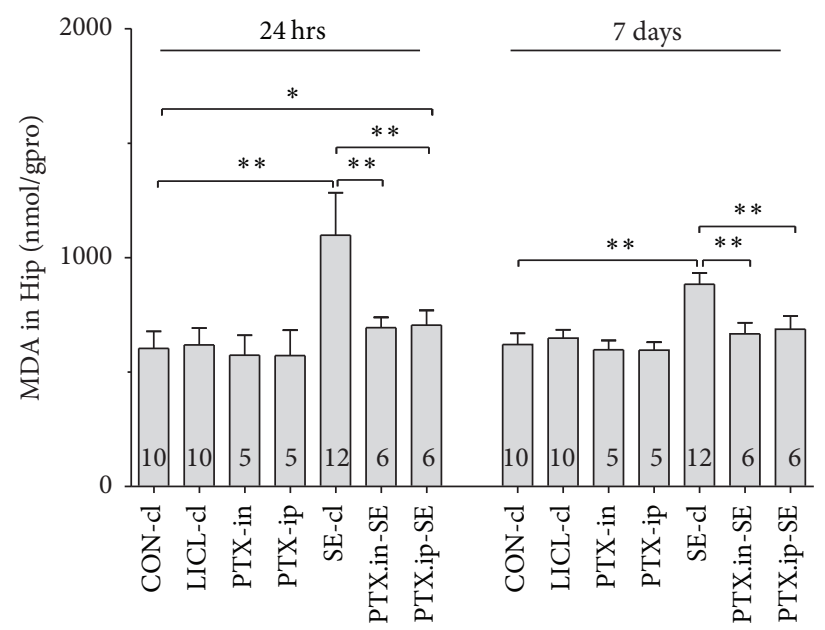

(d)

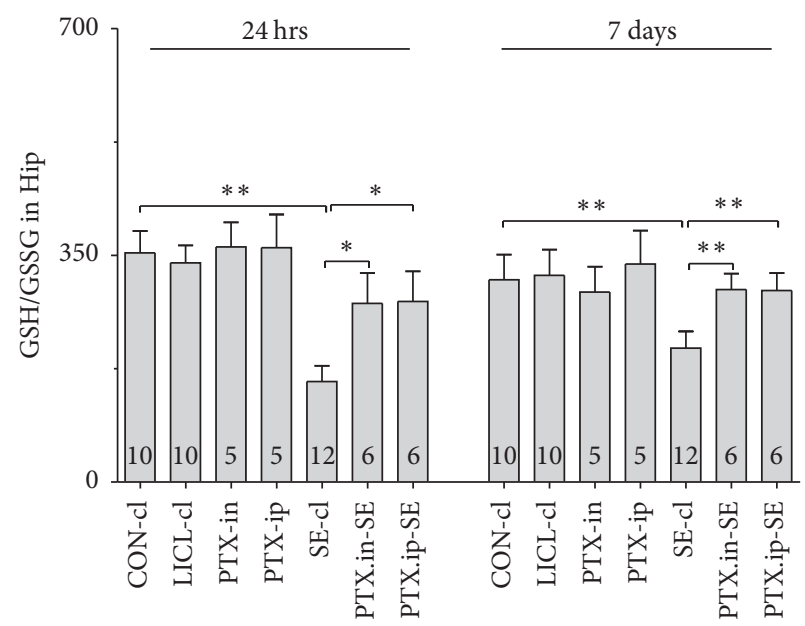

(f)

Figure 4: Effects of intranasal and intraperitoneal Ptx pretreatment on oxidative damage in SN-VTA and Hip of SE rats induced by Li-Pc $24 \mathrm{hrs}$ and 7 days after Pc injection. (a) LPO in SN-VTA. (b) LPO in Hip. (c) MDA in SN-VTA. (d) MDA in Hip. (e) GSH/GSSG in SN-VTA. (f) GSH/GSSG in Hip. The results were expressed as the means \pm SD. ${ }^{*} P<0.05 ;{ }^{* *} P<0.01$. 


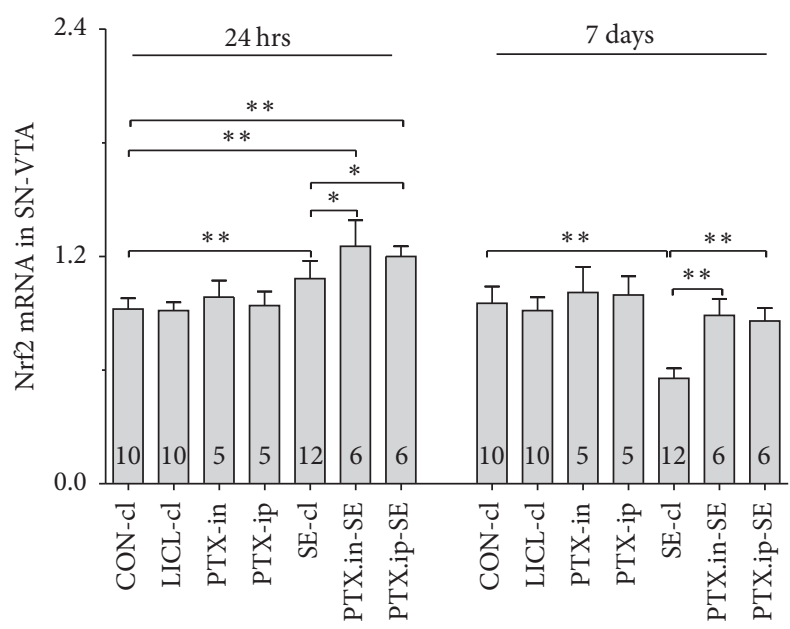

(a)

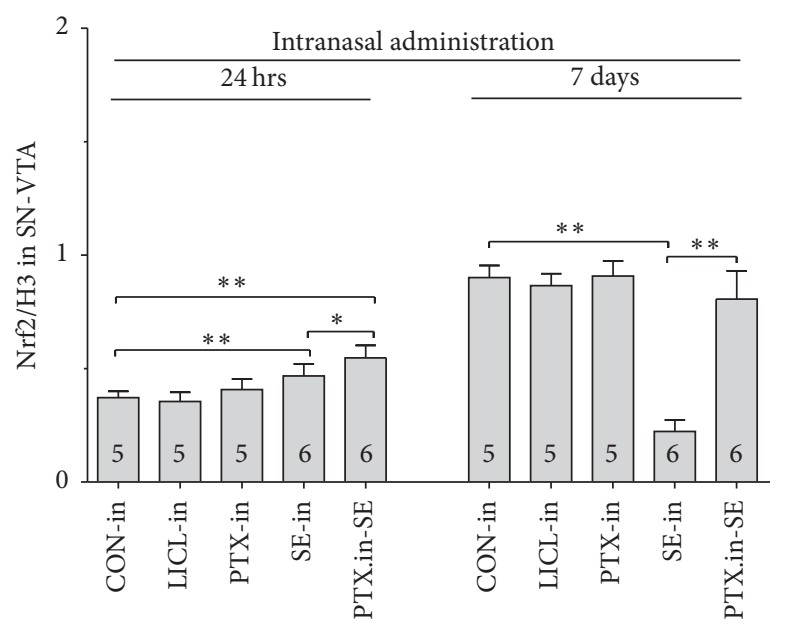

(c)

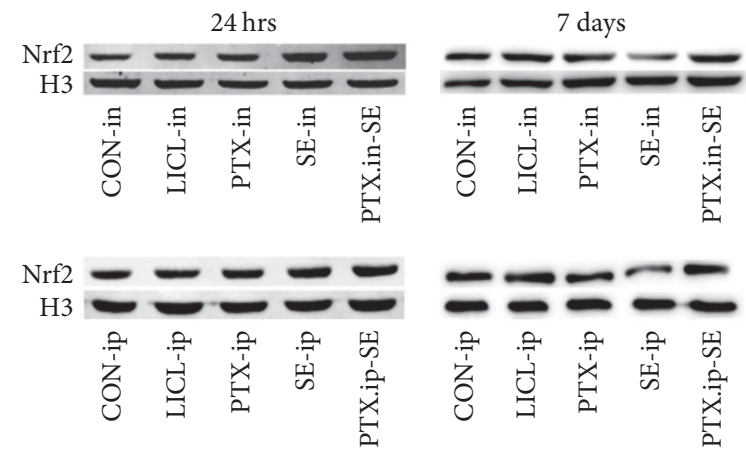

(b)

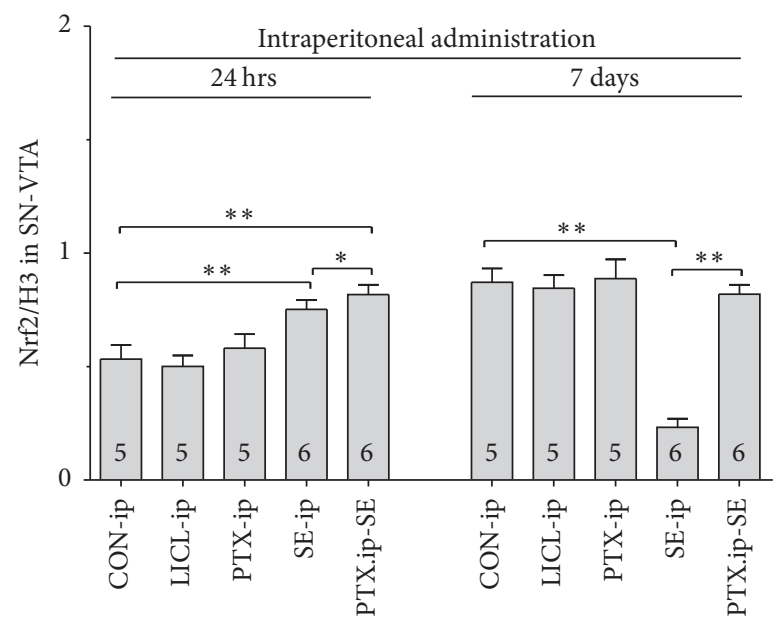

(d)

FIGURE 5: Effects of intranasal and intraperitoneal Ptx pretreatment on Nrf2 in SN-VTA of SE rats induced by Li-Pc 24 hrs and 7 days after Pc injection. (a) Nrf2 mRNA was detected by qPCR. (b-d) Nrf2 protein was measured by Western blot. The results were expressed as the means $\pm \mathrm{SD} .{ }^{*} P<0.05 ;{ }^{* *} P<0.01$.

8(d), Hip; $P<0.01$ ), HO-1 (Figures 6(b) and 6(d), SN-VTA; Figures 9(b) and 9(d), Hip; $P<0.01$ ), and NQO-1 (Figures 7(b) and 7(d), SN-VTA; Figures 10(b) and 10(d), Hip; $P<$ 0.01) among CON-ip, LICL-ip, PTX-ip, SE-ip, and PTX.ip$\mathrm{SE}$ rats. The post hoc test showed that the expression of Nrf2, HO-1, and NQO-1 significantly increased in SN-VTA and in Hip of SE-in and SE-ip rats $24 \mathrm{hrs}$ following Pc injection $(P<0.05)$ and then significantly decreased 7 days after $P c$ administration $(P<0.01)$, compared with corresponding control rats, respectively. In SN-VTA, Ptx pretreatment either by intranasal or intraperitoneal administration to SE rats elevated the levels of Nrf2, HO-1, and NQO-1 $(P<0.05)$. In Hip, except Nrf2 in PTX.in-SE $(P<0.05)$, the increased levels of Nrf2, HO-1, and NQO-1 were not found in PTX.in-SE and in PTX.ip-SE rats $24 \mathrm{hrs}$ following Pc treatment, compared with SE rats. Seven days following Pc administration, the levels of Nrf2, HO-1, and NQO-1 of Hip were higher in Ptxpretreated rats than in SE rats $(P<0.05)$. Nrf2, HO-1, and
NQO-1 were involved in the attenuation of oxidative damage by intranasal Ptx pretreatment to SE rats.

\section{Discussion}

The present studies revealed that the epileptic seizures, the impaired visuospatial memory, and the defected mesodopaminergic system in SE rats induced by $\mathrm{Li}-\mathrm{Pc}$ were effectively ameliorated by pretreatment of Ptx via intranasal delivery, as well as via intraperitoneal injection. Ptx pretreatment decreased the levels of LPO and MDA, increased the ratio of GSH/GSSG, and enhanced the transient activation of $\mathrm{Nrf} 2$ in SE rats. The enhanced transient activation of $\mathrm{Nrf} 2$ was followed by the upregulated HO-1 and NQO-1 in Ptx-pretreated SE rats. Compared with intraperitoneal injection, the poor visuospatial memory and the reduced levels of DA and its metabolites as well as $\mathrm{TH}$ and DAT were completely restored to normal levels 


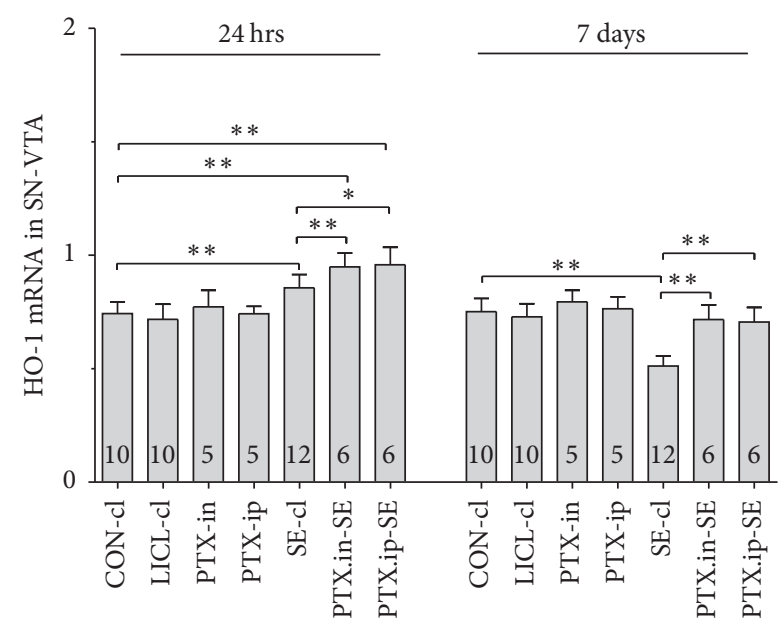

(a)

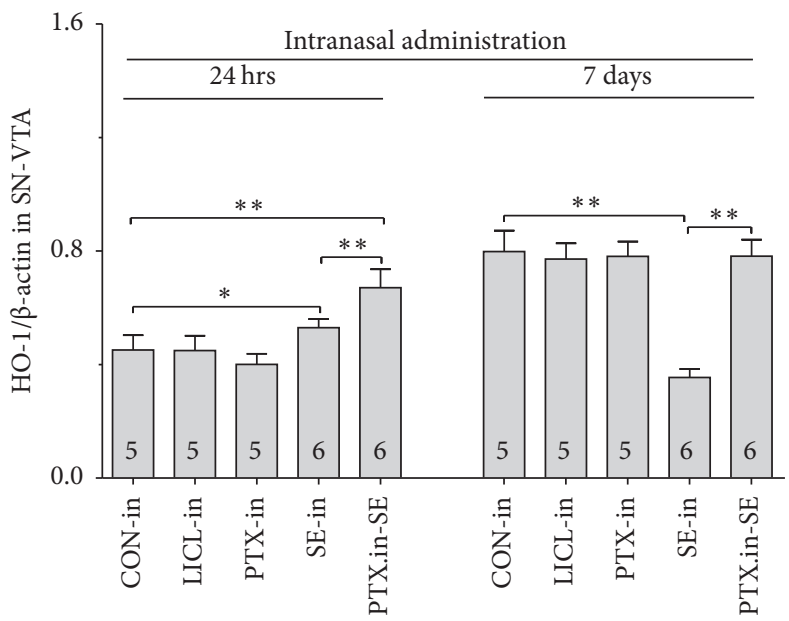

(c)

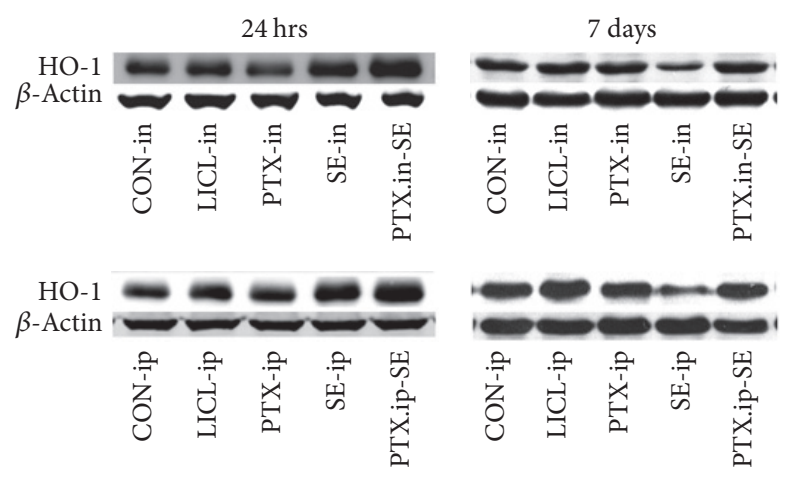

(b)

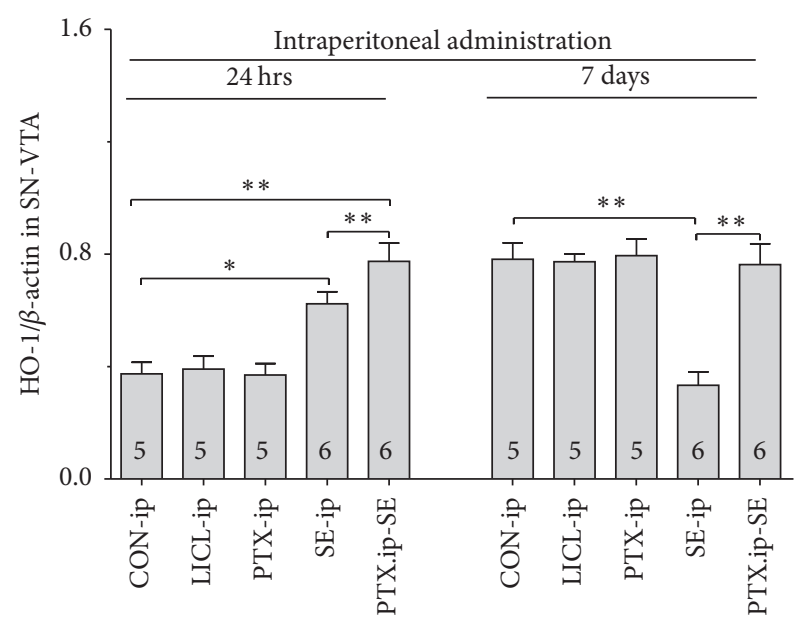

(d)

FIGURE 6: Effects of intranasal and intraperitoneal Ptx pretreatment on HO-1 in SN-VTA of SE rats induced by Li-Pc 24 hrs and 7 days after Pc injection. (a) HO-1 mRNA was detected by qPCR. (b-d) HO-1 protein was measured by Western blot. The results were expressed as the means \pm SD. ${ }^{*} P<0.05 ;{ }^{* *} P<0.01$.

by intranasal pretreatment of Ptx to SE rats. The results above demonstrated the amelioration of epileptic seizures, the alleviation of oxidative damage, and the involvement of Nrf2-ARE pathway in SE rats pretreated by intranasal Ptx.

Lithium-pilocarpine is used to induce SE to reproduce the most features of human SE $[25,26]$. The SE rodent model induced by $\mathrm{Li}-\mathrm{Pc}$ is one of the suitable experimental models in analyzing the pathophysiology of SE $[25,26,34,35]$. Pilocarpine alone at higher doses not only results in a greater likelihood of induction of SE but also increases mortality rate $[36,37]$; however, lithium chloride preadministered within 24 hrs effectively potentiates the epileptogenic action of Pc and simultaneously reduces the mortality of animals $[38,39]$. The previous study found that, 30 minutes prior to induction of SE, intraperitoneal injection of Ptx at the dose of $60 \mathrm{mg} / \mathrm{kg}$ ameliorated the epileptic seizures best among the dose of 0 , 20, 40, and $60 \mathrm{mg} / \mathrm{kg}$ [26]. Thus, $20 \mathrm{mg} / \mathrm{kg} \mathrm{Pc}$ and $60 \mathrm{mg} / \mathrm{kg}$ Ptx were used in the present studies. Consistent with the previous studies $[25,26]$, it was found that all the rats treated by
Li-Pc alone exhibited SE, the impaired visuospatial memory, the defected mesodopaminergic system, and the oxidative damage. Intranasal Ptx pretreatment to SE rats significantly delayed the epileptic seizures, ameliorated the deficits in visuospatial memory, restored the mesodopaminergic function, and attenuated the oxidative stress, which indicated that Ptx pretreatment to SE rats had neuroprotective effects.

Oxidative stress is one of the major factors detrimental to neurons in seizures $[16,40]$. Epileptic seizures decrease the antioxidant capability and increase oxidative damage $[16,40$, 41]. Redox status is a sensitive index of intracellular oxidative stress. GSH/GSSG is a biomarker of redox status in biological systems. We analyzed the ratio of GSH/GSSG by measuring GSH and GSSG in SN-VTA and Hip. GSH, the reduced form of glutathione, was significantly decreased and GSSG, the oxidized form of glutathione, was increased in SN-VTA and Hip of SE rats. GSH and GSSG are redox couples. Glutathione peroxidase catalyzes the reduction of two peroxide molecules using GSH to produce GSSG and water [42]. The diminution 


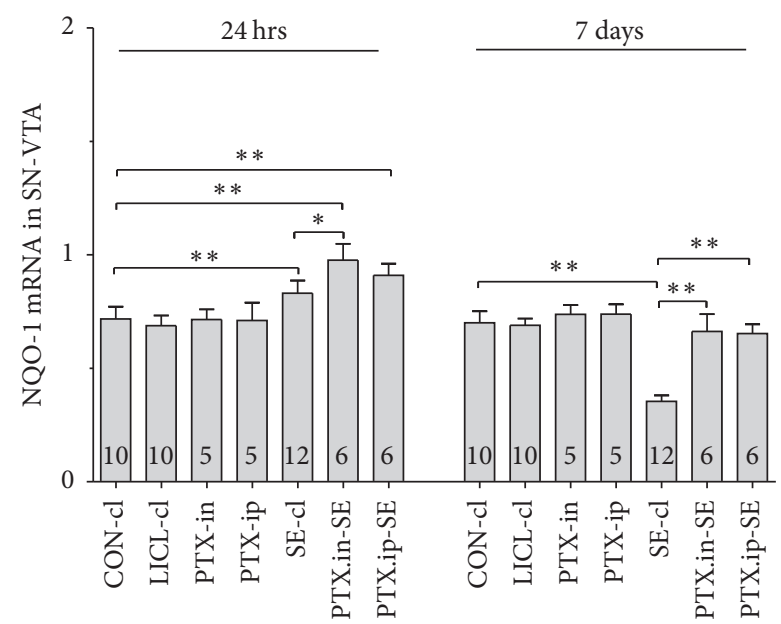

(a)

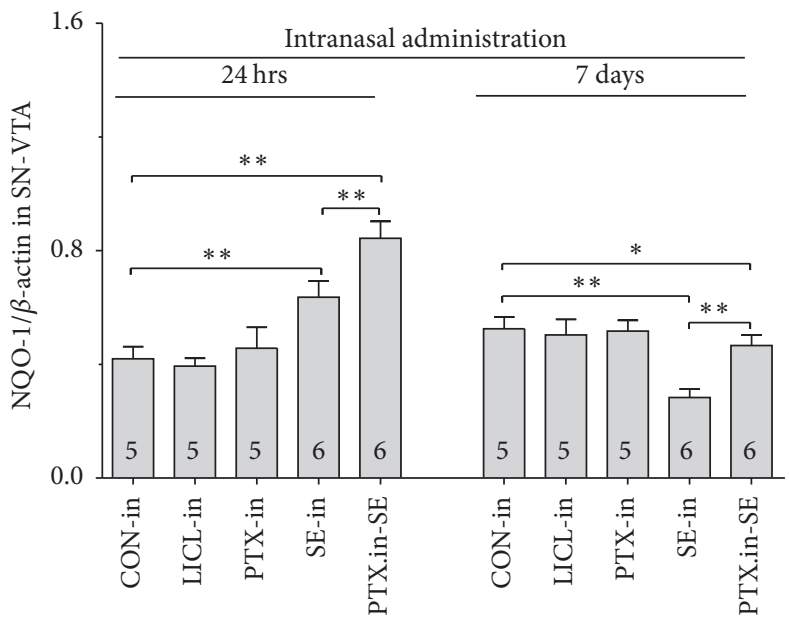

(c)

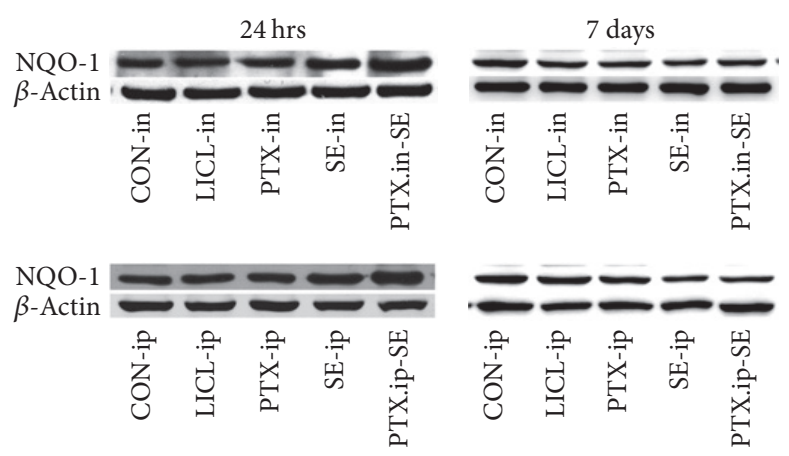

(b)

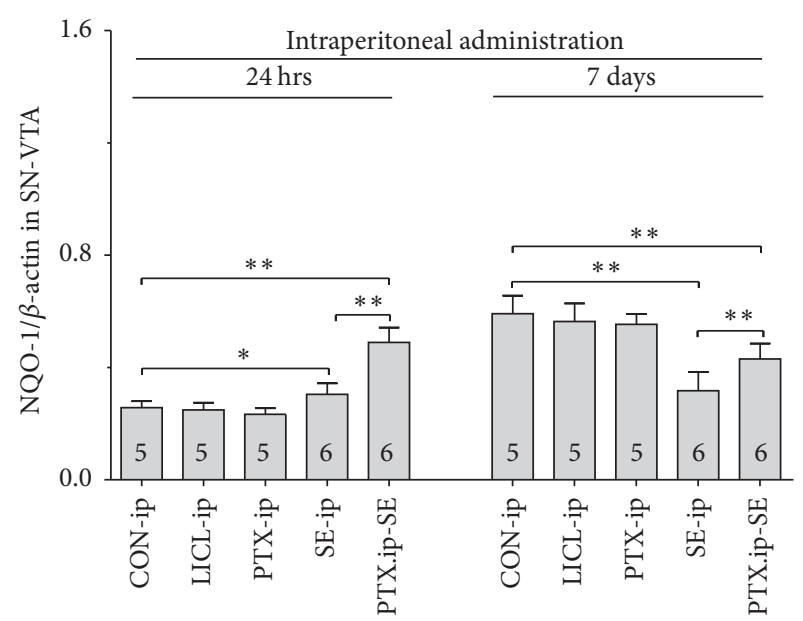

(d)

FIGURE 7: Effects of intranasal and intraperitoneal Ptx pretreatment on NQO-1 in SN-VTA of SE rats induced by Li-Pc 24 hrs and 7 days after Pc injection. (a-c) NQO-1 mRNA was detected by qPCR. (b-d) NQO-1 protein was measured by Western blot. The results were expressed as the means \pm SD. ${ }^{*} P<0.05 ;{ }^{* *} P<0.01$.

of GSH and elevation of GSSG as well as the decreased ratio of GSH/GSSG demonstrated the changes of redox status in SNVTA and Hip of SE rats. Furthermore, two important lipid peroxidation markers, LPO and MDA, were detected in our studies. LPO and MDA are the products of the peroxidation of lipoproteins and phospholipids of biological membranes [43]. The present studies detected the high levels of LPO and MDA in SN-VTA and Hip of SE rats, which suggested that there was oxidative damage to cells in these brain regions of SE rats. Oxidative damage to cells in both Hip and SNVTA might underlie the poor visuospatial memory of SE rats. Compared with SE rats, the reduced LPO and MDA as well as the increased ratio of GSH/GSSG suggested the higher antioxidative ability in SN-VTA and in Hip of PTX.in-SE rats. Increased antioxidative ability might be related to Nrf2-ARE pathway, one of important antioxidative defense systems in suppressing oxidative damage to neurons [44-47].

$\mathrm{Nrf} 2$ is a transcription factor and constitutes the main oxidative stress response in cells $[13,48]$. Under the physiology condition, Nrf2 is located in the cytoplasm. When Nrf2 is activated, it is translocated into the nucleus and combined with the ARE to induce a series of cytoprotective enzymes such as HO-1 [49, 50] and NQO-1 [51, 52] to enhance the antioxidant capacity of cells and protect cells from the oxidative injury $[53,54]$. In the present studies, the elevation of Nrf2, HO-1, and NQO-1 at mRNA and protein levels found in SE rats $24 \mathrm{hrs}$ but not 7 days following Li-Pc injection indicated that epileptic seizures could transiently activate Nrf2-ARE pathway [55]. The transient activation of Nrf2-ARE pathway might be a response to the oxidative stress caused by SE [55], but SE-induced Nrf2 activation was not enough to protect neurons from oxidative damage, which underlay the defects in mesodopaminergic system, as well as the elevated LPO and MDA in SN-VTA and Hip of SE rats in the present studies. However, Nrf2-ARE pathway provides a potential target in controlling epileptic seizures $[24,55]$.

The relevance of Nrf2 in mesolimbic dopaminergic system has not been fully studied; however, it is known that 


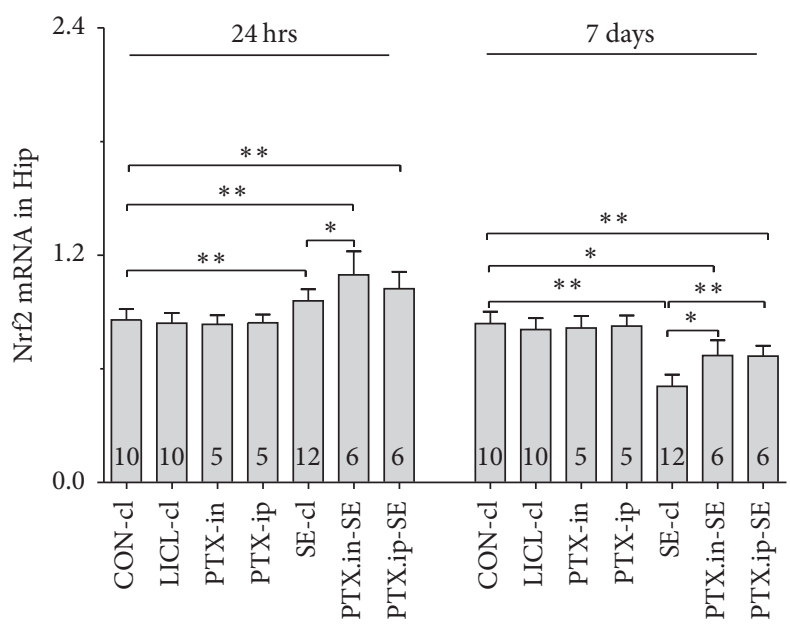

(a)

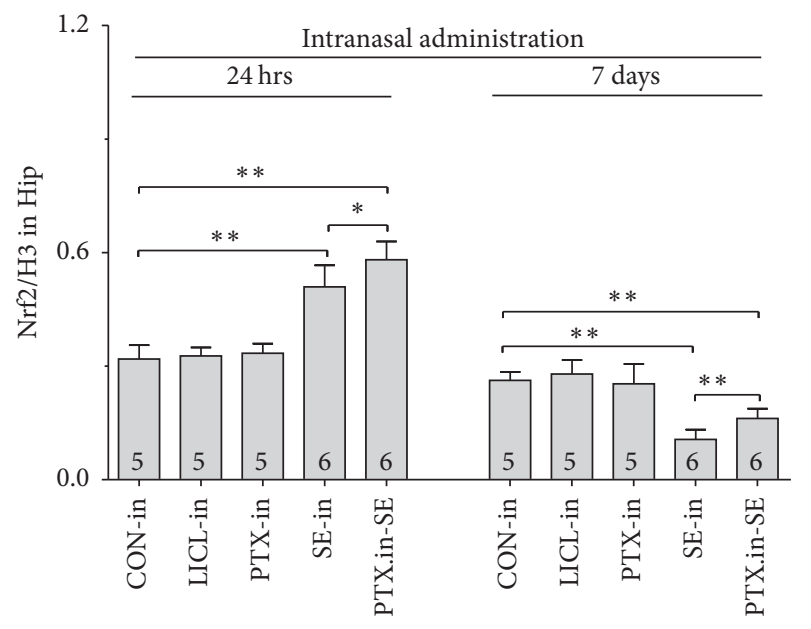

(c)

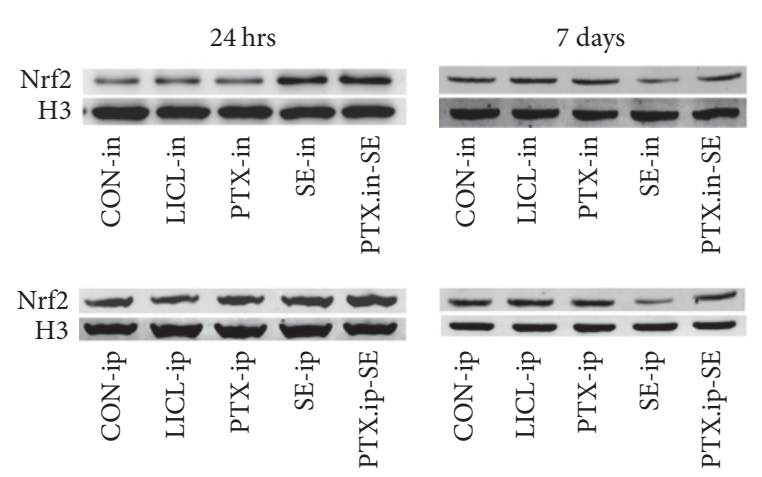

(b)

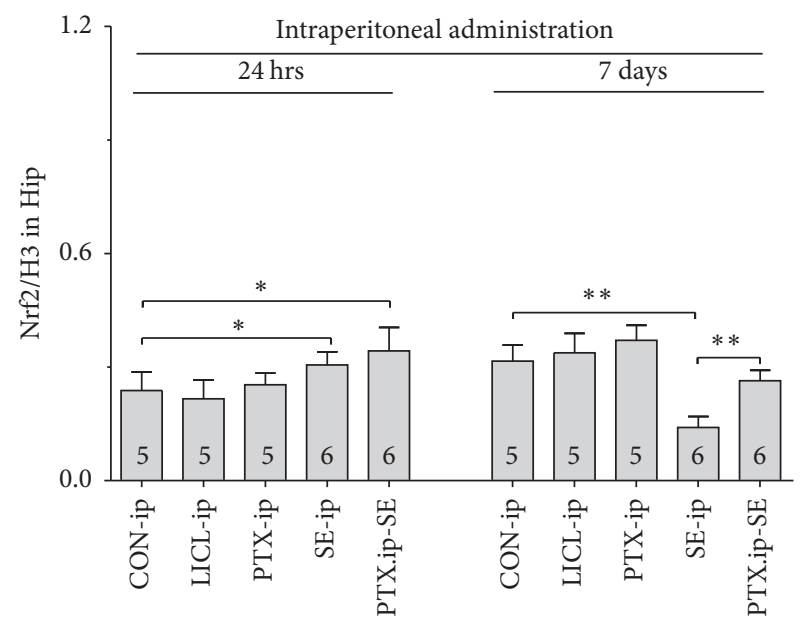

(d)

FIGURE 8: Effects of intranasal and intraperitoneal Ptx pretreatment on Nrf2 in Hip of SE rats induced by Li-Pc 24 hrs and 7 days after Pc injection. (a) Nrf2 mRNA was detected by qPCR. (b-d) Nrf2 protein was measured by Western blot. The results were expressed as the means $\pm \mathrm{SD} .{ }^{*} P<0.05 ;{ }^{* *} P<0.01$.

Nrf2 plays more important role in maintaining normal nigral dopaminergic activity and protects the nigral dopaminergic neurons from neurodegeneration by reducing oxidative stress in Parkinson's disease [56-58]. Activation of Nrf2ARE pathway reduces MPTP-induced neurotoxicity and the death of nigral dopaminergic neurons to a certain extent $[46,57]$. Nigral dopaminergic neurons are more susceptible to MPTP-induced damage in Nrf2-/- mice than $\mathrm{Nrf} 2+/+$ mice [56-58]. Compared with SE rats, the significant elevation of Nrf2, HO-1, and NQO-1 in SN-VTA and Nrf2 in Hip of intranasal Ptx-pretreated SE rats $24 \mathrm{hrs}$ following Pc injection indicated the region-specific involvement of Nrf2-ARE pathway. The following reasons might account for the discrepancy between SN-VTA and Hip. The first might be the fact that Nrf2 differed between various neuronal subpopulations and regulated different gene products in the nigral neurons versus hippocampal neurons [59]. The second reason might be related to DA. The neurotransmitter DA itself can be a source of oxidative stress. Prior to SE, dopaminergic neurons themselves experience the oxidative stimulus due to autooxidation of DA $[60,61]$.

Pentoxifylline, as a nonspecific phosphodiesterase inhibitor, might exert its pharmacological effects during the acute phase of SE by decreasing inflammatory cytokine production $[62,63]$. Moreover, based on our observations on rats treated by Ptx alone, Ptx did not work as Nrf2 activator, which was consistent with the results published by Ahmed and El-Awdan [64]. Administration of Ptx to rats did not alter Nrf2 expression [64]. Evidences demonstrate that the protective role of Nrf2-ARE pathway is mediated by HO-1 and NQO-1 [24]. Compared with control rats or SE rats $24 \mathrm{hrs}$ following Pc, we found that intranasal Ptx pretreatment to SE rats significantly increased the levels of Nrf2, followed by upregulation of HO-1 and NQO-1 at mRNA and protein levels in Ptx-pretreated SE rats, which suggested that intranasal administration of Ptx might increase the antioxidative capability of cells by enhancing the SE-induced transient activation of Nrf2. Due to the fact that 


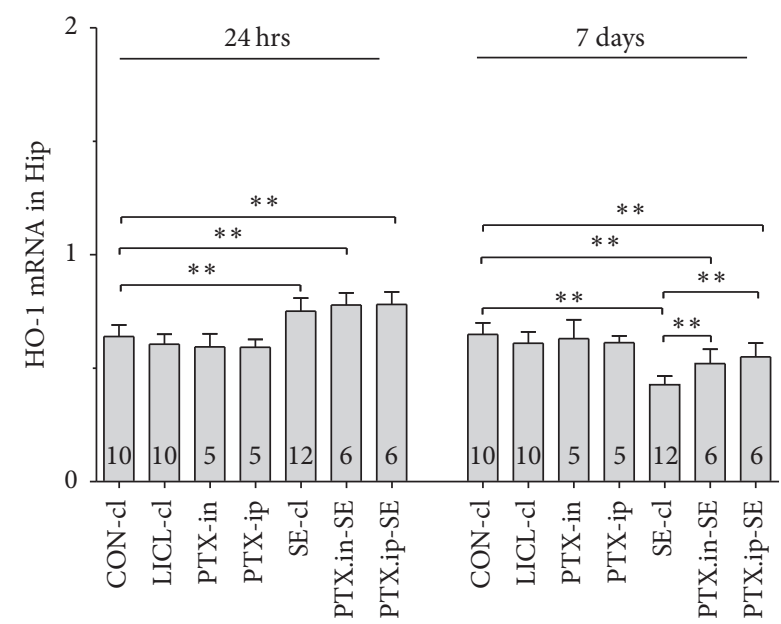

(a)

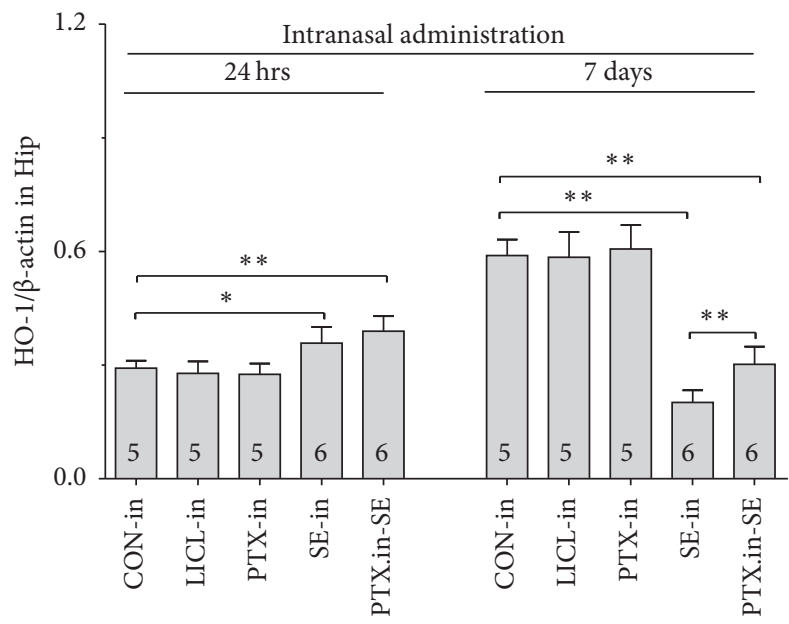

(c)

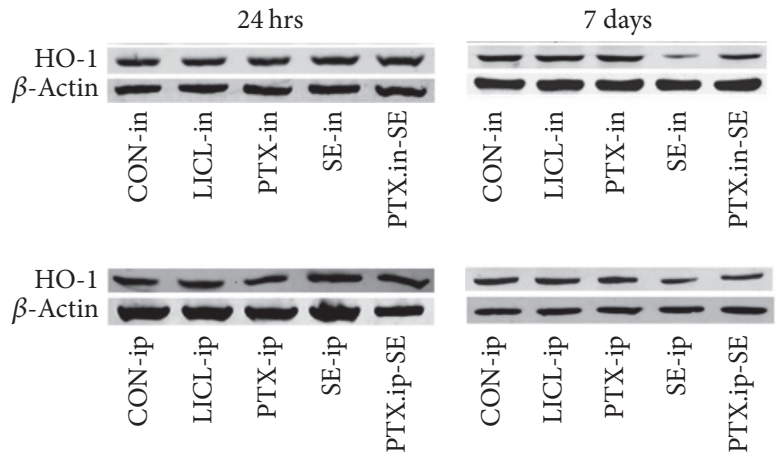

(b)

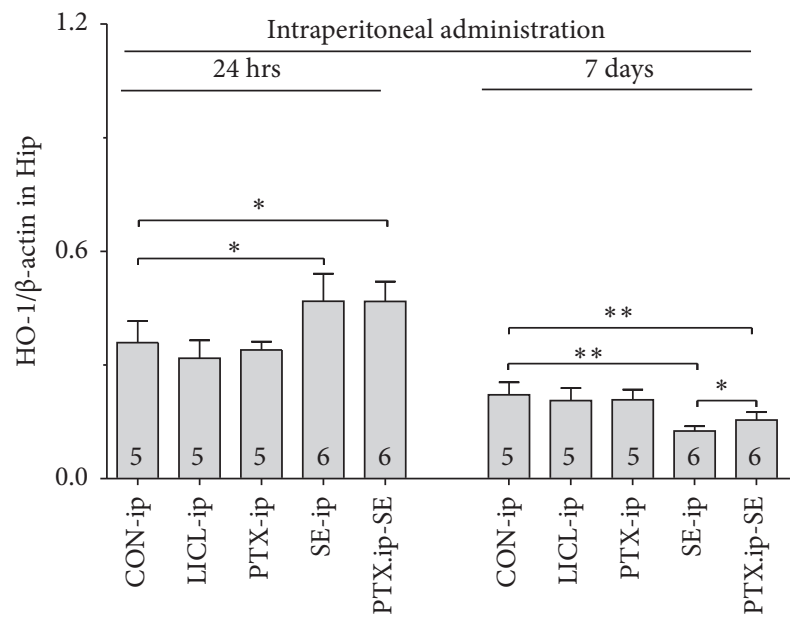

(d)

Figure 9: Effects of intranasal and intraperitoneal Ptx pretreatment on HO-1 in Hip of SE rats induced by Li-Pc 24 hrs and 7 days after Pc injection. (a) HO-1 mRNA was detected by qPCR. (b-d) HO-1 protein was measured by Western blot. The results were expressed as the means $\pm \mathrm{SD} .{ }^{*} P<0.05 ;{ }^{* *} P<0.01$.

the half-life of Nrf2 is very short [65] and at $24 \mathrm{hrs}$ from SE it could be degraded, the time points we choose were $24 \mathrm{hrs}$ and 7 days after administration of Pc as they encompassed both the acute and latent epileptic conditions [66]. How Nrf2 was enhanced in SE rats by Ptx pretreatment might not be determined $24 \mathrm{hrs}$ following Pc injection. Earlier time points in Nrf2 induction of Ptx-pretreated SE rats following Pc injection are necessary to actually unravel if Ptx is cooperating with SE to induce Nrf2 in the future studies.

The large surface area of the nasal mucosa and the abundant blood supply of the nasal cavity make intranasal administration a viable option for delivery of diverse therapeutic compounds $[67,68]$. The unique anatomical connection between the nasal cavity and the brain provides direct noseto-brain delivery of drugs to target the brain through pathways along the olfactory and trigeminal nerves innervating the nasal cavity $[5,7]$. The restoration of impaired visuospatial memory and mesodopaminergic activity in PTX.in-SE rats indicated that intranasal administration of Ptx effectively targets the brain. Compared with PTX.ip-SE rats, the parameters in visuospatial memory and mesodopaminergic system observed in PTX.in-SE rats were completely recovered to the level of control rats 7 days after Pc injection; however, the partial parameters in visuospatial memory and mesodopaminergic system were still lower in PTX.ip-SE rats than in controls. Intranasal administration of Ptx seemed more efficient in controlling SE than intraperitoneal injection. In addition to nose-to-blood-to-brain route (an extensive nasal absorption of the Ptx to the bloodstream), direct nose-tobrain route might be involved in targeting a substantial fraction of Ptx to the brain. Furthermore, Ptx administered intranasally might be reaching the brain expectedly much earlier than through intraperitoneal administration prior to coming insults. Whether a direct transport of Ptx was occurring from nose to the brain, Ptx concentration levels in different cerebral regions and the corresponding rostralto-caudal biodistribution patterns should be determined following both routes of administration in the future studies. 


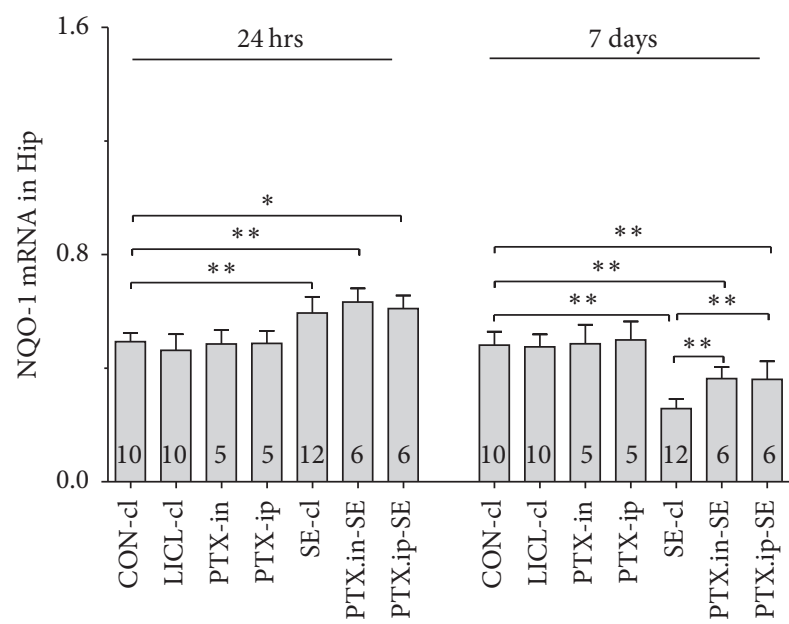

(a)

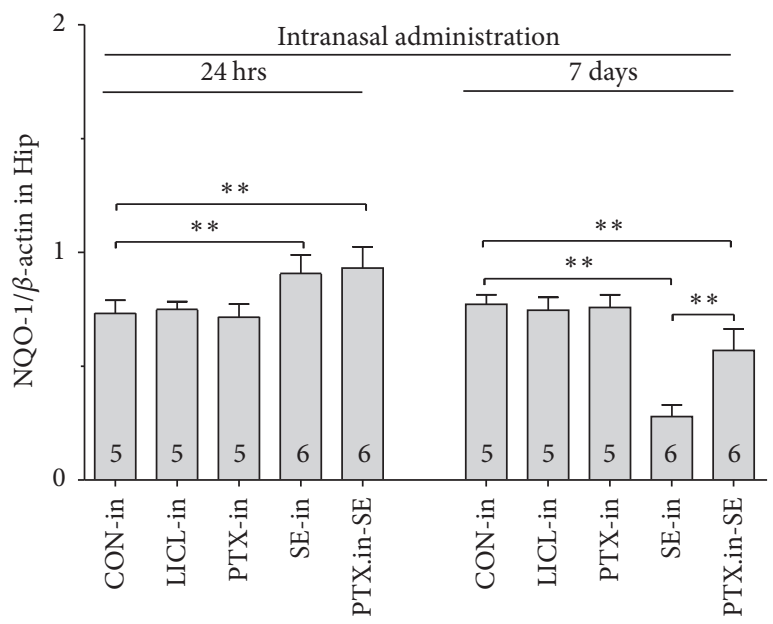

(c)

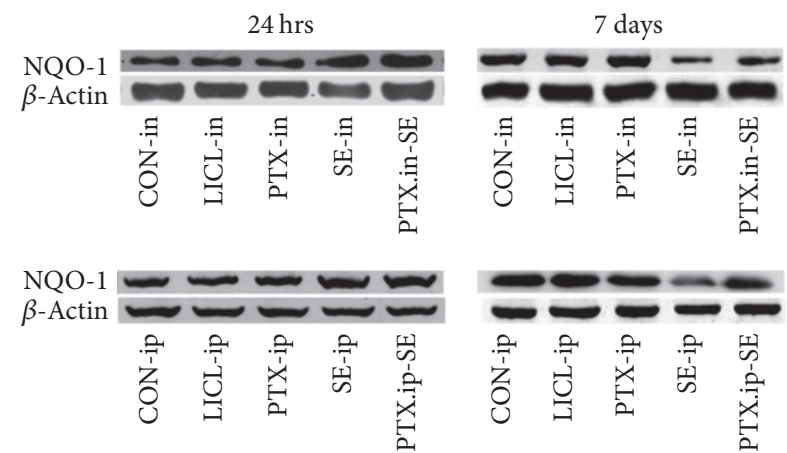

(b)

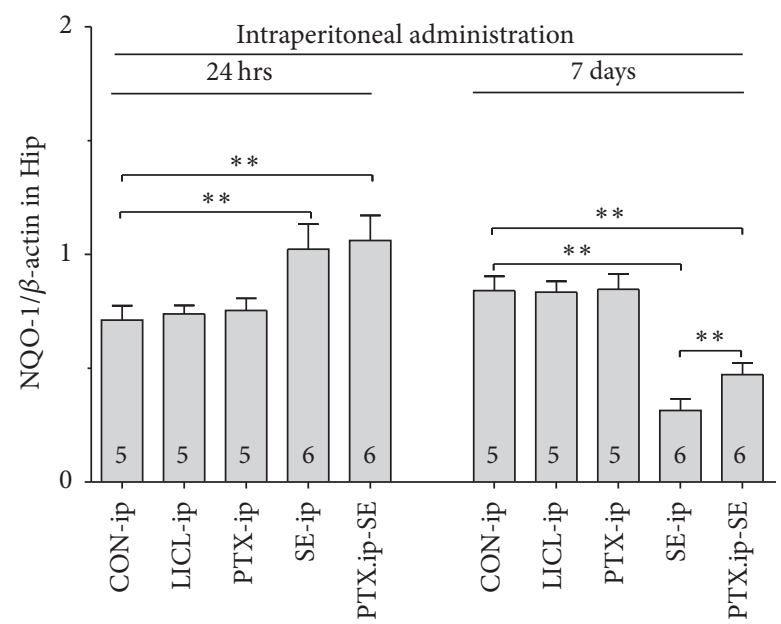

(d)

FIgure 10: Effects of intranasal and intraperitoneal Ptx pretreatment on NQO-1 in Hip of SE rats induced by Li-Pc 24 hrs and 7 days after Pc injection. $(\mathrm{a}-\mathrm{c})$ NQO-1 mRNA was detected by qPCR. (b-d) NQO-1 protein was measured by Western blot. The results were expressed as the means \pm SD. ${ }^{*} P<0.05 ;{ }^{* *} P<0.01$.

In conclusion, intranasal delivery of Ptx to rats significantly suppressed the epileptic seizures induced by Li-Pc, ameliorated the deficits in visuospatial memory and in mesodopaminergic system, and enhanced the transient activation of Nrf2 in SE rats. Intranasal administration of Ptx could effectively protect cells from oxidative damage in SE and may hopefully become a noninvasive, painless, and easily administered option for epileptic patients.

\section{Conflicts of Interest}

The authors declare that there are no conflicts of interest.

\section{Authors' Contributions}

Yunxiao Kang and Wensheng Yan contributed equally to this work.

\section{Acknowledgments}

This work was supported by the Science and Technology Department of Hebei Province of China (Grant no. 12277728) and by Hebei Medical University (Grant no. kyfz022).

\section{References}

[1] F. Al-Mufti and J. Claassen, "Neurocritical care: status epilepticus review," Critical Care Clinics, vol. 30, no. 4, pp. 751-764, 2014.

[2] M. Ahmad, "Protective effects of curcumin against lithiumpilocarpine induced status epilepticus, cognitive dysfunction and oxidative stress in young rats," Saudi Journal of Biological Sciences, vol. 20, no. 2, pp. 155-162, 2013.

[3] R. Kälviäinen, "Intranasal therapies for acute seizures," Epilepsy and Behavior, vol. 49, pp. 303-306, 2015.

[4] M. Zelcer and R. D. Goldman, "Intranasal midazolam for seizure cessation in the community setting," Canadian Family Physician, vol. 62, no. 7, pp. 559-561, 2016. 
[5] J. J. Lochhead and R. G. Thorne, "Intranasal delivery of biologics to the central nervous system," Advanced Drug Delivery Reviews, vol. 64, no. 7, pp. 614-628, 2012.

[6] A. Serralheiro, G. Alves, A. Fortuna, and A. Falcão, "Intranasal administration of carbamazepine to mice: a direct delivery pathway for brain targeting," European Journal of Pharmaceutical Sciences, vol. 60, pp. 32-39, 2014.

[7] A. Serralheiro, G. Alves, A. Fortuna, and A. Falcão, "Direct nose-to-brain delivery of lamotrigine following intranasal administration to mice," International Journal of Pharmaceutics, vol. 490, no. 1-2, pp. 39-46, 2015.

[8] M. Anderson, P. Tambe, H. Sammons, H. Mulla, R. Cole, and I. Choonara, "Pharmacokinetics of buccal and intranasal lorazepam in healthy adult volunteers," European Journal of Clinical Pharmacology, vol. 68, no. 2, pp. 155-159, 2012.

[9] M. Holsti, B. L. Sill, S. D. Firth, F. M. Filloux, S. M. Joyce, and R. A. Furnival, "Prehospital intranasal midazolam for the treatment of pediatric seizures," Pediatric Emergency Care, vol. 23, no. 3, pp. 148-153, 2007.

[10] J. Folbergrová, P. Ješina, N. Kubová, R. Druga, and J. Otáhal, "Status epilepticus in immature rats is associated with oxidative stress and mitochondrial dysfunction," Frontiers in Cellular Neuroscience, vol. 10, article 136, 2016.

[11] V. Mishra, B. Shuai, M. Kodali et al., "Resveratrol treatment after status epilepticus restrains neurodegeneration and abnormal neurogenesis with suppression of oxidative stress and inflammation," Scientific Reports, vol. 5, Article ID 17807, 2015.

[12] J. N. Pearson, S. Rowley, L.-P. Liang, A. M. White, B. J. Day, and M. Patel, "Reactive oxygen species mediate cognitive deficits in experimental temporal lobe epilepsy," Neurobiology of Disease, vol. 82, pp. 289-297, 2015.

[13] L. Carmona-Aparicio, C. Pérez-Cruz, C. Zavala-Tecuapetla et al., "Overview of Nrf2 as therapeutic target in epilepsy," International Journal of Molecular Sciences, vol. 16, no. 8, pp. 18348-18367, 2015.

[14] K. J. Davies, M. E. Delsignore, and S. W. Lin, "Protein damage and degradation by oxygen radicals. II. Modification of amino acids," Journal of Biological Chemistry, vol. 262, no. 20, pp. $9902-$ 9907, 1987.

[15] L. Packer and E. Cadenas, "Oxidants and antioxidants revisited. New concepts of oxidative stress," Free Radical Research, vol. 41, no. 9, pp. 951-952, 2007.

[16] R. M. Freitas, S. M. M. Vasconcelos, F. C. F. Souza, G. S. B. Viana, and M. M. F. Fonteles, "Oxidative stress in the hippocampus after pilocarpine-induced status epilepticus in Wistar rats," FEBS Journal, vol. 272, no. 6, pp. 1307-1312, 2005.

[17] M. Lafon-Cazal, S. Pietri, M. Culcasi, and J. Bockaert, "NMDAdependent superoxide production and neurotoxicity," Nature, vol. 364, no. 6437, pp. 535-537, 1993.

[18] E. K. Michaelis, "Molecular biology of glutamate receptors in the central nervous system and their role in excitotoxicity, oxidative stress and aging," Progress in Neurobiology, vol. 54, no. 4, pp. 369-415, 1998.

[19] N. Basar, L. Nahar, O. A. Oridupa et al., "Utilization of the ability to induce activation of the nuclear factor (erythroid-derived 2)like factor 2 (Nrf2) to assess potential cancer chemopreventive activity of liquorice samples," Phytochemical Analysis, vol. 27, no. 5, pp. 233-238, 2016.

[20] D. M. Miller, I. N. Singh, J. A. Wang, and E. D. Hall, "Nrf2-ARE activator carnosic acid decreases mitochondrial dysfunction, oxidative damage and neuronal cytoskeletal degradation following traumatic brain injury in mice," Experimental Neurology, vol. 264, pp. 103-110, 2015.

[21] M.-K. Kwak, K. Itoh, M. Yamamoto, and T. W. Kensler, "Enhanced expression of the transcription factor Nrf2 by cancer chemopreventive agents: role of antioxidant response elementlike sequences in the nrf2 promoter," Molecular and Cellular Biology, vol. 22, no. 9, pp. 2883-2892, 2002.

[22] A. S. Ahmad, H. Zhuang, and S. Doré, "Heme oxygenase-1 protects brain from acute excitotoxicity," Neuroscience, vol. 141, no. 4, pp. 1703-1708, 2006.

[23] G. Chen, Q. Fang, J. Zhang, D. Zhou, and Z. Wang, "Role of the Nrf2-ARE pathway in early brain injury after experimental subarachnoid hemorrhage," Journal of Neuroscience Research, vol. 89, no. 4, pp. 515-523, 2011.

[24] W. Wang, Y. Wu, G. Zhang et al., "Activation of Nrf2-ARE signal pathway protects the brain from damage induced by epileptic seizure," Brain Research, vol. 1544, pp. 54-61, 2014.

[25] M. Ahmad, G. M. Abu-Taweel, A. E. Aboshaiqah, and J. S. Ajarem, "The effects of quinacrine, proglumide, and pentoxifylline on seizure activity, cognitive deficit, and oxidative stress in rat lithium-pilocarpine model of status epilepticus," Oxidative Medicine and Cellular Longevity, vol. 2014, Article ID 630509, 11 pages, 2014.

[26] M. Tariq, M. Ahmad, K. A. Moutaery, and S. A. Deeb, "Pentoxifylline ameliorates lithium-pilocarpine induced status epilepticus in young rats," Epilepsy and Behavior, vol. 12, no. 3, pp. 354$365,2008$.

[27] Y. Bozzi and E. Borrelli, "The role of dopamine signaling in epileptogenesis," Frontiers in Cellular Neuroscience, vol. 7, article no. 157, 2013.

[28] R. M. De Freitas, F. C. F. De Sousa, S. M. M. Vasconcelos, G. S. B. Viana, and M. M. D. F. Fonteles, "Acute alterations of neurotransmitters levels in striatum of young rat after pilocarpineinduced status epilepticus," Arquivos de Neuro-Psiquiatria, vol. 61, no. 2B, pp. 430-433, 2003.

[29] C. Ciumas, T.-B. R. Wahlin, A. Jucaite, P. Lindstrom, C. Halldin, and I. Savic, "Reduced dopamine transporter binding in patients with juvenile myoclonic epilepsy," Neurology, vol. 71, no. 11, pp. 788-794, 2008.

[30] I. Odano, A. Varrone, I. Savic et al., "Quantitative PET analyses of regional [11C]PE2I binding to the dopamine transporterapplication to juvenile myoclonic epilepsy," NeuroImage, vol. 59, no. 4, pp. 3582-3593, 2012.

[31] A. I. Rojo, C. Montero, M. Salazar et al., "Persistent penetration of MPTP through the nasal route induces Parkinson's disease in mice," European Journal of Neuroscience, vol. 24, no. 7, pp. 18741884, 2006.

[32] R. J. Racine, "Modification of seizure activity by electrical stimulation: II. Motor seizure," Electroencephalography and Clinical Neurophysiology, vol. 32, no. 3, pp. 281-294, 1972.

[33] R. R. González, R. F. Fernández, J. L. M. Vidal, A. G. Frenich, and M. L. G. Pérez, "Development and validation of an ultra-high performance liquid chromatography-tandem massspectrometry (UHPLC-MS/MS) method for the simultaneous determination of neurotransmitters in rat brain samples," Journal of Neuroscience Methods, vol. 198, no. 2, pp. 187-194, 2011.

[34] G. Curia, D. Longo, G. Biagini, R. S. G. Jones, and M. Avoli, "The pilocarpine model of temporal lobe epilepsy," Journal of Neuroscience Methods, vol. 172, no. 2, pp. 143-157, 2008.

[35] C. Dubé, S. Boyet, C. Marescaux, and A. Nehlig, "Relationship between neuronal loss and interictal glucose metabolism during 
the chronic phase of the lithium-pilocarpine model of epilepsy in the immature and adult rat," Experimental Neurology, vol. 167, no. 2, pp. 227-241, 2001.

[36] D. B. Clifford, J. W. Olney, A. Maniotis, R. C. Collins, and C. F. Zorumski, "The functional anatomy and pathology of lithiumpilocarpine and high-dose pilocarpine seizures," Neuroscience, vol. 23, no. 3, pp. 953-968, 1987.

[37] L. Zhao, T. Nagao, G. C. Desjardins, P. Gloor, and M. Avoli, "Quantitative evaluation of neuronal loss in the dorsal hippocampus in rats with long-term pilocarpine seizures," Epilepsy Research, vol. 17, no. 3, pp. 237-247, 1994.

[38] R. Druga, H. Kubová, L. Suchomelová, and R. Haugvicová, "Lithium/pilocarpine status epilepticus-induced neuropathology of piriform cortex and adjoining structures in rats is agedependent," Physiological Research, vol. 52, no. 2, pp. 251-264, 2003.

[39] H. Kubová, P. Mareš, L. Suchomelová, G. Brožek, R. Druga, and A. Pitkänen, "Status epilepticus in immature rats leads to behavioural and cognitive impairment and epileptogenesis," European Journal of Neuroscience, vol. 19, no. 12, pp. 3255-3265, 2004.

[40] B. Martinc, I. Grabnar, and T. Vovk, "Antioxidants as a preventive treatment for epileptic process: a review of the current status," Current Neuropharmacology, vol. 12, no. 6, pp. 527-550, 2014.

[41] T. C. Diniz, J. C. Silva, S. R. G. de Lima-Saraiva et al., "The role of flavonoids on oxidative stress in epilepsy," Oxidative Medicine and Cellular Longevity, vol. 2015, Article ID 171756, 9 pages, 2015.

[42] N. Cardenas-Rodriguez, B. Huerta-Gertrudis, L. RiveraEspinosa et al., "Role of oxidative stress in refractory epilepsy: evidence in patients and experimental models," International Journal of Molecular Sciences, vol. 14, no. 1, pp. 1455-1476, 2013.

[43] H. F. Poon, V. Calabrese, G. Scapagnini, and D. A. Butterfield, "Free radicals and brain aging," Clinics in Geriatric Medicine, vol. 20, no. 2, pp. 329-359, 2004.

[44] N. G. Innamorato, A. Jazwa, A. I. Rojo et al., "Different susceptibility to the parkinson's toxin MPTP in mice lacking the redox master regulator Nrf2 or its target gene heme oxygenase1," PLoS ONE, vol. 5, no. 7, Article ID el1838, 2010.

[45] H. Kumar, S. Koppula, I.-S. Kim, S. V. More, B.-W. Kim, and D.K. Choi, "Nuclear factor erythroid 2 - related factor 2 signaling in Parkinson disease: a promising multi therapeutic target against oxidative stress, neuroinflammation and cell death," CNS and Neurological Disorders-Drug Targets, vol. 11, no. 8, pp. 1015-1029, 2012.

[46] Y.-H. Tsou, C.-T. Shih, C.-H. Ching et al., "Treadmill exercise activates Nrf2 antioxidant system to protect the nigrostriatal dopaminergic neurons from MPP+ toxicity," Experimental Neurology, vol. 263, pp. 50-62, 2015.

[47] G. Zhang, S. Li, Y. Kang et al., "Enhancement of dopaminergic activity and region-specific activation of Nrf2-ARE pathway by intranasal supplements of testosterone propionate in aged male rats," Hormones and Behavior, vol. 80, pp. 103-116, 2016.

[48] H. Zhang, K. J. A. Davies, and H. J. Forman, "Oxidative stress response and Nrf2 signaling in aging," Free Radical Biology and Medicine, vol. 88, pp. 314-336, 2015.

[49] T. Ishii, K. Itoh, S. Takahashi et al., "Transcription factor Nrf2 coordinately regulates a group of oxidative stress-inducible genes in macrophages," Journal of Biological Chemistry, vol. 275, no. 21, pp. 16023-16029, 2000.
[50] F. A. D. T. G. Wagener, H. E. Van Beurden, J. W. Von den Hoff, G. J. Adema, and C. G. Figdor, "The heme-heme oxygenase system: a molecular switch in wound healing," Blood, vol. 102, no. 2, pp. 521-528, 2003.

[51] K. Iskander, J. Li, S. Han, B. Zheng, and A. K. Jaiswal, "NQO1 and NQO2 regulation of humoral immunity and autoimmunity," Journal of Biological Chemistry, vol. 281, no. 41, pp. 3091730924, 2006.

[52] A. K. Jaiswal, "Regulation of genes encoding $\mathrm{NAD}(\mathrm{P}) \mathrm{H}$ : quinone oxidoreductases," Free Radical Biology and Medicine, vol. 29, no. 3-4, pp. 254-262, 2000.

[53] Z. Dong, H. Shang, Y. Q. Chen, L. Pan, M. Bhatia, and J. Sun, "Sulforaphane protects pancreatic acinar cell injury by modulating Nrf2-mediated oxidative stress and NLRP3 inflammatory pathway," Oxidative Medicine and Cellular Longevity, vol. 2016, Article ID 7864150, 12 pages, 2016.

[54] K. Takayama, H. Kaneko, K. Kataoka et al., "Nuclear factor (Erythroid-Derived)-related factor 2-associated retinal pigment epithelial cell protection under blue light-induced oxidative stress," Oxidative Medicine and Cellular Longevity, vol. 2016, Article ID 8694641, 9 pages, 2016.

[55] W. Wang, W.-P. Wang, G.-L. Zhang et al., "Activation of Nrf2ARE signal pathway in hippocampus of amygdala kindling rats," Neuroscience Letters, vol. 543, pp. 58-63, 2013.

[56] P.-C. Chen, M. R. Vargas, A. K. Pani et al., "Nrf2-mediated neuroprotection in the MPTP mouse model of Parkinson's disease: critical role for the astrocyte," Proceedings of the National Academy of Sciences of the United States of America, vol. 106, no. 8, pp. 2933-2938, 2009.

[57] A. Jazwa, A. I. Rojo, N. G. Innamorato, M. Hesse, J. FernándezRuiz, and A. Cuadrado, "Pharmacological targeting of the transcription factor NRf2 at the basal ganglia provides disease modifying therapy for experimental parkinsonism," Antioxidants and Redox Signaling, vol. 14, no. 12, pp. 2347-2360, 2011.

[58] A. I. Rojo, N. G. Innamorato, A. M. Martín-Moreno, M. L. De Ceballos, M. Yamamoto, and A. Cuadrado, "Nrf2 regulates microglial dynamics and neuroinflammation in experimental Parkinson's disease," GLIA, vol. 58, no. 5, pp. 588-598, 2010.

[59] A. D. Kraft, D. A. Johnson, and J. A. Johnson, "Nuclear factor E2-related factor 2-dependent antioxidant response element activation by tert-butylhydroquinone and sulforaphane occurring preferentially in astrocytes conditions neurons against oxidative insult," Journal of Neuroscience, vol. 24, no. 5, pp. 1101$1112,2004$.

[60] B. Fornstedt, E. Pileblad, and A. Carlsson, "In vivo autoxidation of dopamine in guinea pig striatum increases with age," Journal of Neurochemistry, vol. 55, no. 2, pp. 655-659, 1990.

[61] A. Slivka and G. Cohen, "Hydroxyl radical attack on dopamine," Journal of Biological Chemistry, vol. 260, no. 29, pp. 1546615472, 1985.

[62] S. Kwiecień, T. Brzozowski, P. C. Konturek et al., "Gastroprotection by pentoxyfilline against stress-induced gastric damage. Role of lipid peroxidation, antioxidizing enzymes and proinflammatory cytokines," Journal of Physiology and Pharmacology, vol. 55, no. 2, pp. 337-355, 2004.

[63] L. J. Marques, L. Zheng, N. Poulakis, J. Guzman, and U. Costabel, "Pentoxifylline inhibits TNF- $\alpha$ production from human alveolar macrophages," American Journal of Respiratory and Critical Care Medicine, vol. 159, no. 2, pp. 508-511, 1999. 
[64] M. A. E. Ahmed and S. A. El-Awdan, "Lipoic acid and pentoxifylline mitigate nandrolone decanoate-induced neurobehavioral perturbations in rats via re-balance of brain neurotransmitters, up-regulation of Nrf2/HO-1 pathway, and downregulation of TNFR1 expression," Hormones and Behavior, vol. 73, pp. 186-199, 2015.

[65] A. Kobayashi, M.-I. Kang, H. Okawa et al., "Oxidative stress sensor Keap1 functions as an adaptor for Cul3-based E3 ligase to regulate proteasomal degradation of Nrf2," Molecular and Cellular Biology, vol. 24, no. 16, pp. 7130-7139, 2004.

[66] S. Waldbaum, L.-P. Liang, and M. Patel, "Persistent impairment of mitochondrial and tissue redox status during lithiumpilocarpine-induced epileptogenesis," Journal of Neurochemistry, vol. 115, no. 5, pp. 1172-1182, 2010.

[67] A. A. Hussain, "Intranasal drug delivery," Advanced Drug Delivery Reviews, vol. 29, no. 1-2, pp. 39-49, 1998.

[68] A. Pires, A. Fortuna, G. Alves, and A. Falcão, "Intranasal drug delivery: how, why and what for?" Journal of Pharmacy and Pharmaceutical Sciences, vol. 12, no. 3, pp. 288-311, 2009. 


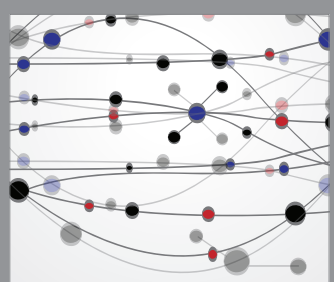

The Scientific World Journal
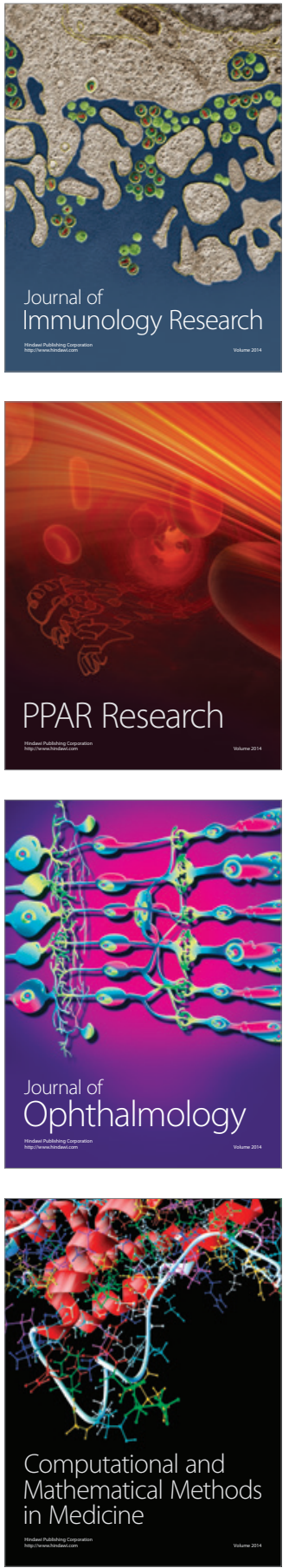

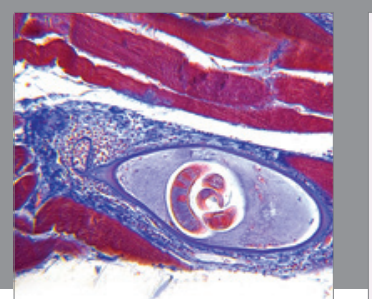

Gastroenterology Research and Practice
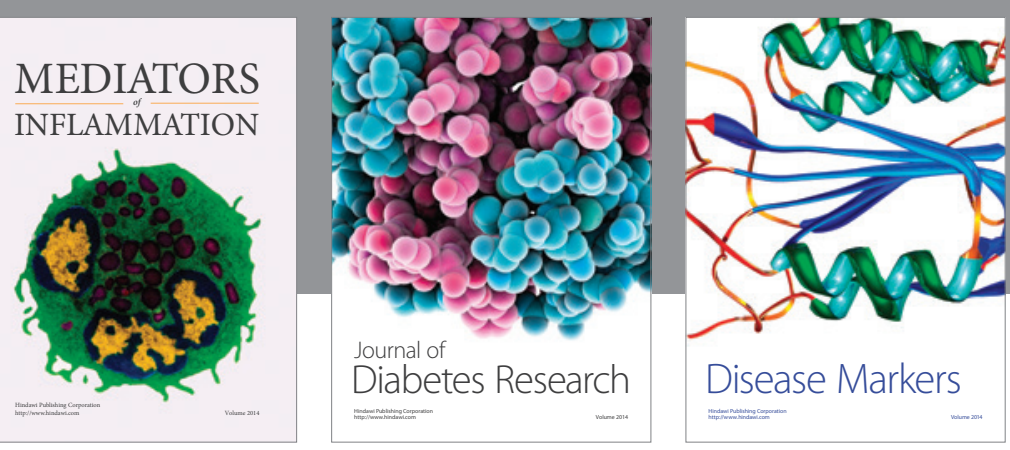

Disease Markers

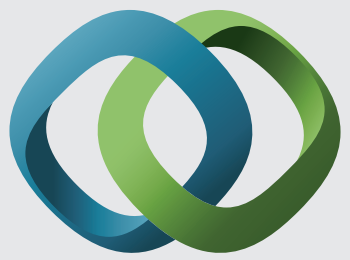

\section{Hindawi}

Submit your manuscripts at

https://www.hindawi.com
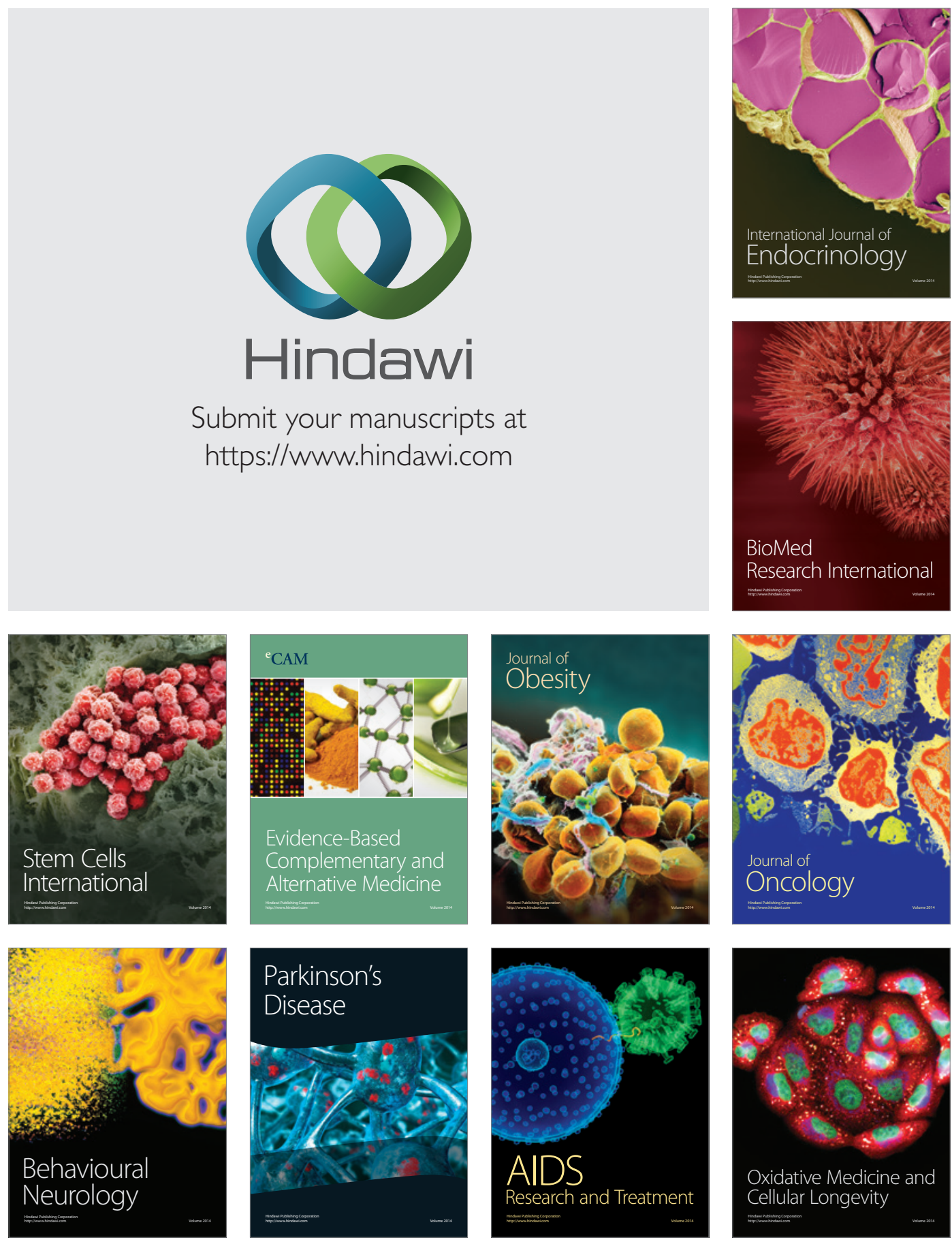